\title{
ATTRACTORS FOR SEMILINEAR DAMPED WAVE EQUATIONS WITH AN ACOUSTIC BOUNDARY CONDITION
}

\author{
Sergio Frigeri \\ Dipartimento di Matematica "F. Casorati" \\ Università di Pavia \\ Via Ferrata, 1 \\ I-27100 Pavia, Italy \\ E-mail address: sergio.frigeri@unipv.it
}

August 5, 2009

\begin{abstract}
In this paper we study a semilinear weakly damped wave equation equipped with an acoustic boundary condition. The problem can be considered as a system consisting of the wave equation describing the evolution of an unknown function $u=u(x, t), x \in \Omega$ in the domain coupled with an ordinary differential equation for an unknown function $\delta=\delta(x, t), x \in \Gamma:=\partial \Omega$ on the boundary. A compatibility condition is also added due to physical reasons. This problem is inspired on a model originally proposed by J. T. Beale and S. I. Rosencrans in [3]. The goal of the paper is to analyze the global asymptotic behavior of the solutions. We prove the existence of an absorbing set and of the global attractor in the energy phase space. Furthermore, the regularity properties of the global attractor are investigated. This is a difficult issue since standard techniques based on the use of fractional operators cannot be exploited. We finally prove the existence of an exponential attractor. The analysis is carried out in dependence of two damping coefficients.
\end{abstract}

\section{Introduction}

Let $\Omega \subset \mathbb{R}^{3}$ be a bounded domain with smooth boundary $\Gamma$. We consider the following semilinear damped wave equation equipped with an acoustic boundary condition

$$
\left\{\begin{array}{lc}
u_{t t}+\omega u_{t}-\Delta u+u+f(u)=0 \quad \text { in } \Omega \times(0, \infty) \\
\delta_{t t}+\nu \delta_{t}+\delta=-u_{t} & \text { on } \Gamma \times(0, \infty) \\
\delta_{t}=\frac{\partial u}{\partial \mathbf{n}} & \text { on } \Gamma \times(0, \infty),
\end{array}\right.
$$


where $\omega$ and $\nu>0$ are an interior and a surface damping parameter, respectively, and the nonlinearity $f \in C^{1}(\mathbb{R})$ fulfills

$$
\begin{aligned}
& \left|f^{\prime}(u)\right| \leq c_{1}\left(1+u^{2}\right), \quad c_{1} \geq 0, \\
& \liminf _{|u| \rightarrow \infty} \frac{f(u)}{u}>-1 .
\end{aligned}
$$

For some results, in place of (1.2) we shall assume that $f \in C^{2}(\mathbb{R})$ satisfies

$$
\begin{gathered}
\left|f^{\prime \prime}(u)\right| \leq c_{2}(1+|u|), \quad c_{2} \geq 0, \\
f^{\prime}(u) \geq-l, \quad l \geq 0 .
\end{gathered}
$$

The physical model described by system (1.1) is that of a gas filling an open bounded domain $\Omega$ whose smooth boundary at each point acts like a spring reacting to the excess pressure of the gas. System (1.1) describes the evolution of the velocity potential $u$ of the fluid which undergoes small perturbations, and of the normal displacement $\delta$ of the boundary into the domain. The model has been considered by J.T. Beale and S. I. Rosencrans in the pioneering paper [3] as a model for acoustic wave motion of a fluid interacting with a so called locally reacting surface, i.e., a surface whose different parts do not interact with each other. See also [25].

Unlike the original model, here we have included a nonlinear term $f(u)$ representing nonlinear effects in the small wave motion of the fluid inside the domain $\Omega$. Moreover, for simplicity, we set equal to 1 all the coefficients in the equations, with the only exception of the damping parameters.

Problem (1.1) (with $f=-u$, i.e., without the nonlinear term and with $\omega=0$ ) has been considered in [3], [4] and in [5] for the exterior domain case. In these papers well-posedness (cf. [4]) has been established and some spectral properties of the evolution semigroup generator ([4], [5]) have been investigated. More recently the interest for acoustic boundary condition has revived. See, for example, [15], [14], [31] and [26], [7], [8].

Though many well-posedness and spectral results have been proved in the existing literature concerning wave equations with acoustic boundary conditions, up to now and to the best of our knowledge, the study of the asymptotic behavior of the solutions is still lacking. Our goal in the present paper is to investigate the long time behavior of system (1.1) focusing the attention on global asymptotic properties, such that the existence of a bounded absorbing set, of the global attractor, of a regular attracting set, as well as of an exponential attractor.

As far as well-posedness and dissipativity are concerned we shall study system (1.1) with a further nonlinear term $g(\delta)$ in the second equation, 
namely

$$
\delta_{t t}+\nu \delta_{t}+\delta+g(\delta)=-u_{t}
$$

where $g \in C^{1}(\mathbb{R})$ fulfills the following assumptions

$$
\begin{aligned}
& \left|g^{\prime}(\delta)\right| \leq c_{3}, \quad c_{3} \geq 0 \\
& \liminf _{|\delta| \rightarrow \infty} \frac{g(\delta)}{\delta}>-1
\end{aligned}
$$

This additional term may represent nonlinear effects in the oscillation of the surface.

The paper is organized as follows. We first conclude this introduction with some essential notation and technical Gronwall type lemmas that we shall use in the course of the investigation. In Section 2 we give the wellposedness result and also a regularity result for the solutions to system (1.1). Due to the particular dynamic boundary condition, the approach that works very well is the semigroup approach. System (1.1) is written as an abstract evolution equation in the energy phase space for a vector unkown whose four components are the velocity potential in the domain $u$ (and its time derivative) and the displacement on the boundary $\delta$ (and its time derivative).

In light of the well-posednesss result, system (1.1) (or system $(1.1)_{1},(1.6)$, $\left.(1.1)_{3}\right)$, associated with an initial condition, is the generator of a dynamical system $\left(\mathcal{H}, S_{\omega, \nu}(t)\right)$ on the phase space $\mathcal{H}$.

In Section 3 we deduce the dissipativity of the system by proving the existence of a bounded absorbing set in the energy phase space. A sufficient condition on the nonlinearities $f$ and $g$ for the uniform decay of the trajectories departing from every bounded subset of $\mathcal{H}$ is also obtained.

Section 4 is devoted to the result on the existence of the global attractor for system (1.1). We wish to obtain this result by means of the same hypotheses used for well-posedness (i.e. (1.2) and (1.3)), without using further assumptions. To this aim, the classical decomposition method does not work very well. Indeed, it can be seen that this method either requires (1.4), (1.5) (see Section 5) or the very restrictive assumption $\left|f^{\prime}(u)\right| \leq c$. The reason is that, due to the dynamic boundary condition, we cannot work here with fractional powers of some elliptic operator in order to deduce the asymptotic compactness by means of estimates in more regular Sobolev spaces. Therefore, a different approach has to be employed and the idea is to use the energy equation method, originally due to J. M. Ball.

In Section 5 we consider the regularity of the attractor. More precisely, we show the existence of a regular bounded set which attracts the bounded subsets of the energy phase space exponentially fast. This is done by employing a decomposition technique which is essentially due to V. Pata and 
S. Zelik (see [28]). The result of Section 5 is also useful for Section 6 which is devoted to the construction of an exponential attractor, by means of the technique devised in [10].

Notation. We denote by $(\cdot, \cdot)$ and $\|\cdot\|$ the inner product and the norm on $L^{2}(\Omega)$, respectively. For every $s \in \mathbb{R}$, the norm in the Sobolev space $H^{s}(\Omega)$ will be denoted by $\|\cdot\|_{s}$. The inner product on $L^{2}(\Gamma)$ is $\langle\cdot, \cdot\rangle$ and the corresponding norm is simply $\|\cdot\|_{L^{2}(\Gamma)}$. The finite energy phase space of the solution semigroup is

$$
\mathcal{H}:=H^{1}(\Omega) \times L^{2}(\Omega) \times L^{2}(\Gamma) \times L^{2}(\Gamma) .
$$

We shall also need the Laplace-Beltrami operator $-\Delta_{\Gamma}$ on the variety $\Gamma$. We recall that $-\Delta_{\Gamma}$ is a positive definite self-adjoint operator in $L^{2}(\Gamma)$, with domain $D\left(-\Delta_{\Gamma}\right)$. The Sobolev spaces $H^{s}(\Gamma)$ on the variety $\Gamma$, for $s \in \mathbb{R}$, can thus be defined as $H^{s}(\Gamma)=D\left(\left(-\Delta_{\Gamma}\right)^{s / 2}\right)$, endowed with the graph norm

$$
\|v\|_{H^{s}(\Gamma)}^{2}=\|v\|_{L^{2}(\Gamma)}^{2}+\left\|\left(-\Delta_{\Gamma}\right)^{s / 2} v\right\|_{L^{2}(\Gamma)}^{2} .
$$

The following lemma will be particularly useful to prove the dissipative feature of the system

Lemma 1. Let $X$ be a Banach space, and $\mathcal{Z} \subset C([0,+\infty) ; X)$. Let there be given a functional $E: X \rightarrow \mathbb{R}$ such that

$$
\sup _{t \geq 0} E(z(t)) \geq-m, \quad E(z(0)) \leq M
$$

for some $m, M \geq 0$ and for every $z \in \mathcal{Z}$. In addition assume that $E(z(\cdot)) \in$ $C^{1}([0,+\infty))$ for every $z \in \mathcal{Z}$ and that the differential inequality

$$
\frac{d}{d t} E(z(t))+\epsilon_{0}\|z(t)\|_{X}^{2} \leq k
$$

holds for all $t \geq 0$ and for some $\epsilon_{0}>0, k \geq 0$, both independent of $z \in \mathcal{Z}$. Then, for every $\eta>0$ there is $t_{0}=t_{0}(M, \eta) \geq 0$ such that, for every $z \in \mathcal{Z}$

$$
E(z(t)) \leq \sup _{\zeta \in X}\left\{E(\zeta): \epsilon_{0}\|\zeta\|_{X}^{2} \leq k+\eta\right\}
$$

for every $t \geq t_{0}$. Furthermore, the time $t_{0}$ can be expressed by $t_{0}=(M+m) / \eta$.

For the proof see, for instance, [6, Lemma 2.7]. Furthermore, in order to prove the main result of Section 5, we shall need two suitable versions of the Gronwall lemma that, though well known, we shall now recall for the reader's convenience (for their proof see [9, Lemma 2.1] and [21, Lemma 2.2], respectively). 
Lemma 2. Let $\Psi:[0,+\infty) \rightarrow[0,+\infty)$ be an absolutely continuous function satisfying

$$
\frac{d}{d t} \Psi(t)+2 \epsilon \Psi(t) \leq h(t) \Psi(t)+k
$$

where $\epsilon>0, k \geq 0$ and $\int_{s}^{t} h(\tau) d \tau \leq \epsilon(t-s)+m$, for all $t \geq s \geq 0$ and some $m \geq 0$. Then

$$
\Psi(t) \leq \Psi(0) e^{m} e^{-\epsilon t}+\frac{k e^{m}}{\epsilon}
$$

for all $t \geq 0$.

Lemma 3. Let $\Phi:[0, \infty) \rightarrow[0, \infty)$ be an absolutely continuous function such that, for some $\epsilon>0$

$$
\frac{d}{d t} \Phi(t)+2 \epsilon \Phi(t) \leq f(t) \Phi(t)+h(t)
$$

for almost every $t \in[0, \infty)$, where $f$ and $h$ are functions on $[0, \infty)$ such that

$$
\int_{s}^{t}|f(\tau)| d \tau \leq \alpha\left(1+(t-s)^{\lambda}\right), \quad \sup _{t \geq 0} \int_{t}^{t+1}|h(\tau)| d \tau \leq \beta
$$

for some $\alpha, \beta \geq 0$ and $\lambda \in[0,1)$. Then

$$
\Phi(t) \leq \gamma \Phi(0) e^{-\epsilon t}+K
$$

for every $t \in[0, \infty)$, for some $\gamma=\gamma(f, \epsilon, \lambda) \geq 1$ and $K=K(\epsilon, \lambda, f, h) \geq 0$. The constants $\gamma, K$ can be given by $\gamma=\exp \left\{\alpha+\alpha(\alpha \lambda / \epsilon)^{\lambda /(1-\lambda)}-\epsilon(\alpha \lambda / \epsilon)^{1 /(1-\lambda)}\right\}$ and $K=\gamma \beta C_{\epsilon}$, where $C_{\epsilon}=e^{\epsilon} /\left(1-e^{-\epsilon}\right)$.

\section{Well-posedness}

The existence of the solution semigroup $S(t)$ for the initial value problem associated with (1.1) has been proved in [4] for the linear case $f=0$. The same proof, which relies on the use of the semigroup method by writing (1.1) in the form of an abstract semilinear evolution equation in $\mathcal{H}$, can be obviously adapted also for the present case with $f$ different from zero.

The initial value problem for (1.1) can be naturally formulated in the finite energy Hilbert space $\mathcal{H}$ endowed with the norm

$$
\|w\|_{\mathcal{H}}^{2}=\left\|w_{1}\right\|_{1}^{2}+\left\|w_{2}\right\|^{2}+\left\|w_{3}\right\|_{L^{2}(\Gamma)}^{2}+\left\|w_{4}\right\|_{L^{2}(\Gamma)}^{2}
$$

for every $w:=\left(w_{1}, w_{2}, w_{3}, w_{4}\right) \in \mathcal{H}$. Actually, it is easy to see that the (linear) energy of a solution $w=\left(u, u_{t}, \delta, \delta_{t}\right)$ to $(1.1)$ is

$$
E_{w}(t)=\frac{1}{2}\|w(t)\|_{\mathcal{H}}^{2}
$$


and the (nonlinear) energy is

$$
\mathcal{E}_{w}(t)=E_{w}(t)+\int_{\Omega} F(u)+\int_{\Gamma} G(\delta),
$$

where $F(s)=\int_{o}^{s} f(\sigma) d \sigma$ and $G(s)=\int_{0}^{s} g(\sigma) d \sigma$, which formally satisfies the energy equation

$$
\frac{d \mathcal{E}_{w}}{d t}=-\omega\left\|u_{t}\right\|^{2}-\nu\left\|\delta_{t}\right\|_{L^{2}(\Gamma)}^{2}
$$

Now, according to [4], we introduce the linear unbouded operator $A: D(A) \subset$ $\mathcal{H} \rightarrow \mathcal{H}$ defined by

$$
\begin{gathered}
D(A)=\left\{w=\left(w_{1}, w_{2}, w_{3}, w_{4}\right) \in \mathcal{H}: \Delta w_{1} \in L^{2}(\Omega), w_{2} \in H^{1}(\Omega), \partial_{\mathbf{n}} w_{1}=w_{4}\right\} \\
A w:=\left(w_{2},-\omega w_{2}+\Delta w_{1}-w_{1}, w_{4},-\left(w_{2}+w_{3}+\nu w_{4}\right)\right)
\end{gathered}
$$

for every $w=\left(w_{1}, w_{2}, w_{3}, w_{4}\right) \in \mathcal{H}$. The condition $\partial_{\mathbf{n}} w_{1}=w_{4}$ is interpreted in the weak sense to mean

$$
\int_{\Omega}\left(\Delta w_{1}\right) \varphi+\nabla w_{1} \cdot \nabla \varphi=\int_{\Gamma} w_{4} \varphi
$$

for every $\varphi \in H^{1}(\Omega)$. Furthermore, we introduce the nonlinear function $\mathcal{F}$ : $\mathcal{H} \rightarrow \mathcal{H}$ defined by

$$
\mathcal{F}(w)=\left(0,-f\left(w_{1}\right), 0,-g\left(w_{3}\right)\right),
$$

for every $w \in \mathcal{H}$. The initial value problem for system $(1.1)_{1},(1.6),(1.1)_{3}$ can therefore be put in the form

$$
\left\{\begin{array}{l}
w_{t}=A w+\mathcal{F}(w) \\
w(0)=w_{0}
\end{array}\right.
$$

where $w=\left(u, u_{t}, \delta, \delta_{t}\right)$ and $w_{0}=\left(u_{0}, u_{1}, \delta_{0}, \delta_{1}\right) \in \mathcal{H}$. In order to consider strong solutions we also introduce the following (second order) phase space

$\mathcal{H}_{1}=\left\{w=\left(w_{1}, w_{2}, w_{3}, w_{4}\right) \in H^{2}(\Omega) \times H^{1}(\Omega) \times H^{1 / 2}(\Gamma) \times H^{1 / 2}(\Gamma): \partial_{\mathbf{n}} w_{1}=w_{4}\right\}$

which is Hilbert with the associated norm

$$
\|w\|_{\mathcal{H}_{1}}^{2}=\left\|w_{1}\right\|_{2}^{2}+\left\|w_{2}\right\|_{1}^{2}+\left\|w_{3}\right\|_{H^{1 / 2}(\Gamma)}^{2}+\left\|w_{4}\right\|_{H^{1 / 2}(\Gamma)}^{2} .
$$

Due to the smoothness of the boundary $\Gamma$ it easy to check that

$$
\mathcal{H}_{1}=D(A) \cap Z
$$


where $Z$ is the subspace

$$
Z=\left\{w=\left(w_{1}, w_{2}, w_{3}, w_{4}\right) \in \mathcal{H}: w_{3}, w_{4} \in H^{1 / 2}(\Gamma)\right\}
$$

It can be seen (cf. [4, Theorem 2.1]) that $A$ is the generator of a strongly continuous contraction semigroup $\left\{e^{A t}\right\}_{t \geq 0}$ of bounded linear operators on $\mathcal{H}$, and $\mathcal{F}$ is a locally Lipschitz map on $\mathcal{H}$. We recall the notion of weak solution to $(2.2)$.

Definition 1. Let $w_{0} \in \mathcal{H}$. We say that $w \in C^{0}([0, \infty) ; \mathcal{H})$ is a weak solution to (2.2) if it satisfies

$$
w(t)=e^{A t} w_{0}+\int_{0}^{t} e^{A(t-s)} \mathcal{F}(w(s)) d s,
$$

for all $t \geq 0$.

Denoting by $A^{*}$ the adjoint of $A$, It can be proved (see [2]) that a map $w \in C^{0}([0, \infty) ; \mathcal{H})$ is a weak solution to $(2.2)$ if and and only if for each $z \in D\left(A^{*}\right)$ the function $(w(\cdot), z)_{\mathcal{H}}$ is absolutely continuous on $[0, T]$, for each $T>0$, and satisfies

$$
\frac{d}{d t}(w(t), z)_{\mathcal{H}}=\left(w(t), A^{*} z\right)_{\mathcal{H}}+(\mathcal{F}(w(t)), z)_{\mathcal{H}}, \quad \text { for a.e. } t \in[0, \infty)
$$

and the initial condition $w(0)=w_{0}$. We now can state the main theorem of this section.

Theorem 1. Let (1.2), (1.3), (1.7), (1.8) hold and assume that $w_{0} \in \mathcal{H}$. Then, there exists a unique weak solution $w \in C^{0}([0, \infty) ; \mathcal{H})$ to $(2.2)$. For each weak solution $\mathcal{E}_{w}(\cdot) \in C^{1}([0, \infty))$ and the energy equation $(2.1)$ holds. Furthermore, if $w_{01}$ and $w_{02}$ are two sets of data in $\mathcal{H}$ and $w_{1}, w_{2}$ the corresponding solutions on $[0, \infty)$, there exists $\theta>0$, depending only on the $\mathcal{H}$-norms of the data and independent of $\omega, \nu$, such that

$$
\left\|w_{2}(t)-w_{1}(t)\right\|_{\mathcal{H}} \leq e^{\theta t}\left\|w_{02}-w_{01}\right\|_{\mathcal{H}}
$$

for all $t \geq 0$. Assuming, in addition, that $f$ fulfills (1.4), that $g=0$, and that $w_{0} \in \mathcal{H}_{1}$, the corresponding weak solution satisfies the regularity property

$$
w \in C^{1}([0, \infty) ; \mathcal{H}) \cap C^{0}\left([0, \infty) ; \mathcal{H}_{1}\right)
$$

and is called "strong" solution. 
Proof. We give a sketch of the proof. The fact that $D(A)$ is dense in $\mathcal{H}$ has been shown in [4, Theorem 2.1], and one can use the same argument as in [4, Theorem 2.1] to prove that $A$ is also closed, dissipative, i.e., $(A w, w)_{\mathcal{H}} \leq 0$, for every $w \in D(A)$, and such that $R(I-A)=\mathcal{H}$. By the Lumer-Phillips Theorem we therefore conclude that $A$ is the generator of a $C^{0}$ contraction semigroup in $\mathcal{H}$.

Due to (1.2) and (1.7) we have that $\mathcal{F}: \mathcal{H} \rightarrow \mathcal{H}$ is locally Lipschitz and so, for every $w_{0} \in \mathcal{H}$ there exists $t_{\max }=t_{\max }\left(w_{0}\right) \in(0, \infty]$ such that (2.2) admits a unique maximally defined weak solution $w \in C^{0}\left(\left[0, t_{\max }\right) ; \mathcal{H}\right)$. To show that $t_{\max }=\infty$ one can use the energy identity (2.1) which can be derived by adapting the argument used by J. M. Ball in [1, Theorem 3.6].

Integrating (2.1) between 0 and $t$,

$$
\begin{aligned}
& \|w(t)\|_{\mathcal{H}}^{2}+2 \int_{\Omega} F(u)+2 \int_{\Gamma} G(\delta)+\int_{0}^{t}\left(\omega\left\|u_{t}\right\|^{2}+\nu\left\|\delta_{t}\right\|_{L^{2}(\Gamma)}^{2}\right) d \tau \\
& =\left\|w_{0}\right\|_{\mathcal{H}}^{2}+2 \int_{\Omega} F\left(u_{0}\right)+2 \int_{\Gamma} G\left(\delta_{0}\right)
\end{aligned}
$$

for every $t \in\left[0, t_{\max }\right)$. By $(1.3)$ and $(1.8)$ there exist $\mu_{0}, \mu_{1} \in(0,1]$ such that

$$
2 \int_{\Omega} F(u) \geq-\left(1-\mu_{0}\right)\|u\|_{1}^{2}-c_{4}
$$

and

$$
2 \int_{\Gamma} G(\delta) \geq-\left(1-\mu_{1}\right)\|\delta\|_{L^{2}(\Gamma)}^{2}-c_{5} .
$$

Plugging (2.6), (2.7) into (2.5) and using also assumptions (1.2) and (1.7), one immediately gets

$$
\|w(t)\|_{\mathcal{H}} \leq C\left(\left\|w_{0}\right\|_{\mathcal{H}}\right)
$$

for every $t \in\left[0, t_{\max }\right)$, and this implies that $t_{\max }=\infty$. Furthermore, we have that, for every $R>0$, there exists a positive constant $C=C(R)$, independent of $\omega$ and $\nu$, such that, whenever $\left\|w_{0}\right\|_{\mathcal{H}} \leq R$, the corresponding solution fulfills

$$
\|w(t)\|_{\mathcal{H}} \leq C
$$

for all $t \geq 0$. For the continuous dependence estimate (2.3), setting $\widetilde{w}:=$ $w_{2}-w_{1}$ and $\widetilde{w}_{0}=w_{02}-w_{01}$, we easily get

$$
\begin{aligned}
& \frac{1}{2} \frac{d}{d t}\|\widetilde{w}\|_{\mathcal{H}}^{2}+\omega\left\|\widetilde{u}_{t}\right\|^{2}+\nu\left\|\widetilde{\delta}_{t}\right\|_{L^{2}(\Gamma)}^{2} \\
& =\left(f\left(u_{1}\right)-f\left(u_{2}\right), \widetilde{u}_{t}\right)+\left\langle g\left(\delta_{1}\right)-g\left(\delta_{2}\right), \widetilde{\delta}_{t}\right\rangle .
\end{aligned}
$$


By (1.2)

$$
\left|\left(f\left(u_{1}\right)-f\left(u_{2}\right), \widetilde{u}_{t}\right)\right| \leq C(R)\|\widetilde{u}\|_{1}\left\|\widetilde{u}_{t}\right\|
$$

where $R>0$ is such that $\left\|w_{0 i}\right\|_{\mathcal{H}} \leq R$, for $i=1,2$, and the second term on the right hand side of (2.9) can be obviously estimated by $c_{2}\|\widetilde{\delta}\|_{L^{2}(\Gamma)}\left\|\widetilde{\delta}_{t}\right\|_{L^{2}(\Gamma)}$, due to (1.7). Hence, (2.3) follows immediately from (2.9) and (2.10), by using the standard Gronwall lemma.

Finally, for the regularity result in the case $g=0$ we can reason in the spirit of $[4$, Lemma 2.3]. We give the main points for the convenience of the reader.

Due to assumption (1.4) on $f$ and to the fact that $g=0$, the map $\mathcal{F}$ : $D(A) \rightarrow D(A)$ is locally Lipschitz continuous. Hence (see, i.e., [33, Theorem 2.5.6.]), if $w_{0} \in \mathcal{H}_{1}$ (which implies $w_{0} \in D(A)$ ), the corresponding weak solution $w$ fulfills

$$
w \in C^{1}([0, \infty) ; \mathcal{H}) \cap C^{0}([0, \infty) ; D(A)) .
$$

Consider now the following initial value problem in $X:=H^{1 / 2}(\Gamma) \times H^{1 / 2}(\Gamma)$

$$
\left\{\begin{array}{l}
z_{t}=B z+y \\
z(0)=\left(\delta_{0}, \delta_{1}\right)^{T}
\end{array}\right.
$$

where $z(t)=\left(z_{3}(t), z_{4}(t)\right)^{T}$ and

$$
B:=\left[\begin{array}{cc}
0 & 1 \\
-1 & -\nu
\end{array}\right], \quad y(t):=\left[\begin{array}{c}
0 \\
-u_{t}(t)
\end{array}\right]
$$

$u_{t}$ being the second component of the solution $w$. Therefore $y \in C^{0}([0, \infty) ; X)$, as a consequence of (2.11). As $B \in \mathcal{L}(X)$ (hence $B$ is the generator of a uniformly continuous $C^{0}$ semigroup on $\left.X\right)$ and $z(0) \in X$, we have that problem (2.12) admits a unique solution $z \in C^{1}([0, \infty) ; X)$. By comparision with problem (2.2) (the two equations in (2.12) are equivalent to the second equation in (2.2)) and by uniqueness, we deduce that $z_{3}(t)=\delta(t)$ and $z_{4}(t)=\delta_{t}(t)$, for every $t \geq 0$. This, together with (2.11), implies (2.4).

Theorem 1 and the fact that the system is autonomous lead immediately to the following.

Corollary 1. In the hypotheses (1.2), (1.3), (1.7) and (1.8), system (1.1) ${ }_{1}$, (1.6), (1.1) $)_{3}$ with the initial condition $w(0)=w_{0} \in \mathcal{H}$ generates a strongly continuous semigroup $S(t)=S_{\omega, \nu}(t)$ on the phase space $\mathcal{H}$. 


\section{Dissipativity}

In this section we show the existence of a bounded absorbing set $\mathcal{B}_{0} \subset \mathcal{H}$ for system $(1.1)_{1},(1.6),(1.1)_{3}$. The analysis is carried out in dependence of the damping coefficients $\omega$ and $\nu$.

We point out that, though the calculations we perform in this and in the subsequent sections are formal, the estimates (and also all the differential identities) can be justified by means of an appropriate regularization procedure which relies on the semigroup theory and on the regularity result of Section 2 (see [13]).

Here is the result we want to prove.

Theorem 2. Let (1.2), (1.3), (1.7), (1.8) hold. Then, there exists $R_{0}>0$ with the following property: for every $R>0$, there exists $t_{0}=t_{0}(R, \omega, \nu)$ such that, for every $w_{0} \in \mathcal{H}$ with $\left\|w_{0}\right\|_{\mathcal{H}} \leq R$, we have

$$
\left\|S(t) w_{0}\right\|_{\mathcal{H}} \leq R_{0}
$$

for every $t \geq t_{0}$.

Proof. We set $\xi=u_{t}+\epsilon u, \zeta=\delta_{t}+\epsilon \delta$, where $\epsilon>0$ is to be fixed later, and we multiply in $L^{2}(\Omega)$ the first equation of system (1.1) by $\xi$ and in $L^{2}(\Gamma)$ the second equation by $\zeta$. Adding the resulting equations we get

$$
\begin{aligned}
& \frac{1}{2} \frac{d}{d t}\left\{\|u\|_{1}^{2}+\|\xi\|^{2}+\|\delta\|_{L^{2}(\Gamma)}^{2}+\|\zeta\|_{L^{2}(\Gamma)}^{2}\right. \\
& \left.+2 \epsilon\langle u, \delta\rangle+2 \int_{\Omega} F(u)+2 \int_{\Gamma} G(\delta)\right\} \\
& +\epsilon\|u\|_{1}^{2}+(\omega-\epsilon)\|\xi\|^{2}-\epsilon(\omega-\epsilon)(u, \xi) \\
& +\epsilon\|\delta\|_{L^{2}(\Gamma)}^{2}+(\nu-\epsilon)\|\zeta\|_{L^{2}(\Gamma)}^{2}-\epsilon(\nu-\epsilon)\langle\delta, \zeta\rangle \\
& +\epsilon(f(u), u)+\epsilon\langle g(\delta), \delta\rangle=2 \epsilon\langle u, \zeta\rangle-2 \epsilon^{2}\langle u, \delta\rangle .
\end{aligned}
$$

Henceforth in this section $c_{i}, k_{1}, k_{2}, \epsilon_{i}$ and $\alpha$ will be some positive (or nonegative) constants whose value will be specified in Remark 2. We now have

$$
\begin{aligned}
& -\epsilon(\omega-\epsilon)(u, \xi) \geq-\frac{\epsilon \mu_{0}}{4}\|u\|_{1}^{2}-\frac{\epsilon \omega^{2}}{\mu_{0}}\|\xi\|^{2} \\
& -\epsilon(\nu-\epsilon)\langle\delta, \zeta\rangle \geq-\frac{\epsilon \mu_{1}}{2}\|\delta\|_{L^{2}(\Gamma)}^{2}-\frac{\epsilon \nu^{2}}{2 \mu_{1}}\|\zeta\|_{L^{2}(\Gamma)}^{2} \\
& \epsilon(f(u), u) \geq-\epsilon\left(1-\mu_{o}\right)\|u\|_{1}^{2}-\epsilon c_{6} \\
& \epsilon\langle g(\delta), \delta\rangle \geq-\epsilon\left(1-\mu_{1}\right)\|\delta\|_{L^{2}(\Gamma)}^{2}-\epsilon c_{7} \\
& 2 \epsilon\langle u, \zeta\rangle \leq \frac{\epsilon \mu_{0}}{4}\|u\|_{1}^{2}+\frac{4 \epsilon c_{t}^{2}}{\mu_{0}}\|\zeta\|_{L^{2}(\Gamma)}^{2} \\
& -2 \epsilon^{2}\langle u, \delta\rangle \leq \epsilon^{2}\|\delta\|_{L^{2}(\Gamma)}^{2}+\epsilon^{2} c_{t}^{2}\|u\|_{1}^{2},
\end{aligned}
$$


where $\mu_{0}$ and $\mu_{1}$ are the same as in (2.6) and (2.7), respectively, and $c_{t}$ is the constant in the trace inequality $\|v\|_{L^{2}(\Gamma)} \leq c_{t}\|v\|_{1}$, for every $v \in H^{1}(\Omega)$. Plugging (3.2)-(3.7) into (3.1) we get

$$
\begin{aligned}
& \frac{1}{2} \frac{d}{d t}\left\{\|u\|_{1}^{2}+\|\xi\|^{2}+\|\delta\|_{L^{2}(\Gamma)}^{2}+\|\zeta\|_{L^{2}(\Gamma)}^{2}\right. \\
& \left.+2 \epsilon\langle u, \delta\rangle+2 \int_{\Omega} F(u)+2 \int_{\Gamma} G(\delta)\right\} \\
& +\frac{\epsilon}{2}\left(\mu_{0}-2 c_{t}^{2} \epsilon\right)\|u\|_{1}^{2}+\left(\omega-\epsilon\left(1+\frac{\omega^{2}}{\mu_{0}}\right)\right)\|\xi\|^{2} \\
& +\frac{\epsilon}{2}\left(\mu_{1}-2 \epsilon\right)\|\delta\|_{L^{2}(\Gamma)}^{2}+\left(\nu-\epsilon\left(1+\frac{4 c_{t}^{2}}{\mu_{0}}+\frac{\nu^{2}}{2 \mu_{1}}\right)\right)\|\zeta\|_{L^{2}(\Gamma)}^{2} \\
& \leq \epsilon\left(c_{6}+c_{7}\right) .
\end{aligned}
$$

Let us now define the functional

$$
\begin{aligned}
& E(w):=\|u\|_{1}^{2}+\|v\|^{2}+\|\delta\|_{L^{2}(\Gamma)}^{2}+\|\eta\|_{L^{2}(\Gamma)}^{2} \\
& +2 \epsilon\langle u, \delta\rangle+2 \int_{\Omega} F(u)+2 \int_{\Gamma} G(\delta)
\end{aligned}
$$

for every $w=(u, v, \delta, \eta) \in \mathcal{H}$. It is easy to see that, by taking $\epsilon \in\left(0, \epsilon_{1}\right]$, where $\epsilon_{1}>0$ is obviously independent of $\omega, \nu$, we have

$$
k_{1}\|w\|_{\mathcal{H}}^{2}-c_{8} \leq E(w) \leq k_{2}\|w\|_{\mathcal{H}}\left(1+\|w\|_{\mathcal{H}}^{3}\right),
$$

for every $w \in \mathcal{H}$, where $k_{1}, k_{2}$ are two positive constants independent of $\omega$, $\nu$, and $c_{8}=c_{4}+c_{5}$. If we now introduce

$$
K(s):=s /\left(1+s^{2}\right),
$$

we see that, choosing $0<\epsilon \leq \min \left\{\epsilon_{2}, \frac{1}{2 \alpha} K(\omega), \frac{1}{2 \alpha} K(\nu)\right\}$, where $\epsilon_{2}>0$ is independent of $\omega, \nu$, we have

$$
\frac{d}{d t} E(w)+\frac{\epsilon}{2} \mu_{0}\|u\|_{1}^{2}+\omega\|\xi\|^{2}+\frac{\epsilon}{2} \mu_{1}\|\delta\|_{L^{2}(\Gamma)}^{2}+\nu\|\zeta\|_{L^{2}(\Gamma)}^{2} \leq \epsilon\left(c_{6}+c_{7}\right) .
$$

Furthermore, for $\epsilon \in(0,1 / 2]$

$$
\|u\|_{1}^{2}+\|\xi\|^{2}+\|\delta\|_{L^{2}(\Gamma)}^{2}+\|\zeta\|_{L^{2}(\Gamma)}^{2} \geq \frac{1}{2}\|w\|_{\mathcal{H}}^{2} .
$$

In order to highlight the dependence on $\omega, \nu$ in this section (see also Section 5) we introduce

$$
J(\omega, \nu):=\min \left\{\epsilon_{1}, \epsilon_{2}, \frac{1}{2}, \frac{\omega}{2 \mu^{*}}, \frac{\nu}{2 \mu^{*}}, \frac{K(\omega)}{2 \alpha}, \frac{K(\nu)}{2 \alpha}\right\},
$$


where $\mu^{*}=\frac{1}{4} \min \left\{\mu_{0}, \mu_{1}\right\}$. Therefore, by taking $\epsilon=J(\omega, \nu)$, from (3.11) and (3.12) we are led to

$$
\frac{d}{d t} E(w(t))+\mu^{*} J(\omega, \nu)\|w(t)\|_{\mathcal{H}}^{2} \leq\left(c_{6}+c_{7}\right) J(\omega, \nu) .
$$

The theorem now follows by applying Lemma 1. Indeed, let us fix $R>0$ and a set of initial data $w_{0} \in \mathcal{H}$ such that $\left\|w_{0}\right\|_{\mathcal{H}} \leq R$. By (3.10) we have the bound

$$
E(w(0)) \leq k_{2} R\left(1+R^{3}\right)
$$

From Lemma 1, we deduce that there exists a time $t_{0}=t_{0}(R, \omega, \nu)>0$ such that

$$
E\left(S(t) w_{0}\right) \leq \sup \left\{E(z): z \in \mathcal{H}, \quad \mu^{*} J(\omega, \nu)\|z\|_{\mathcal{H}}^{2} \leq 2\left(c_{6}+c_{7}\right) J(\omega, \nu)\right\},
$$

for every $t \geq t_{0}$ and for every $w_{0} \in \mathcal{H}$ with $\left\|w_{0}\right\|_{\mathcal{H}} \leq R$. Hence, on account of (3.10) we conclude that there exists $R_{0}>0$, independent of $\omega, \nu$, such that

$$
\left\|S(t) w_{0}\right\|_{\mathcal{H}} \leq R_{0}
$$

for every $t \geq t_{0}$ and every $w_{0} \in \mathcal{H}$ such that $\left\|w_{0}\right\|_{\mathcal{H}} \leq R$. The ball

$$
\mathcal{B}_{0}=\left\{z \in \mathcal{H}:\|z\|_{\mathcal{H}} \leq R_{0}\right\}
$$

is therefore a bounded absorbing set for the semigroup. Moreover, the time $t_{0}(R, \omega, \nu)$ can be expressed by $t_{0}(R, \omega, \nu)=\left(k_{2} R\left(1+R^{3}\right)+c_{8}\right) /\left(c_{6}+c_{7}\right) J(\omega, \nu)$.

Remark 1. We observe that the radius of the absorbing set $R_{0}$ does not depend on the damping coefficients $\omega, \nu$. Nevertheless, the time $t_{0}$ needed to stabilize the system not only depends increasingly on $R$, as expected, but depends also on $\omega$ and $\nu$. In particular we point out that, for fixed $R>0$, we have $t_{0} \rightarrow \infty$ both for $\omega$ or $\nu \rightarrow 0$ and for $\omega$ or $\nu \rightarrow \infty$.

Physically, the $\omega, \nu$-dependence of $t_{0}$ is obvious in the former case, as in this case the dissipation is reduced to zero. On the other hand, in the latter case, a very large damping has the effect of freezing the system, since the damping acts only on the velocities $u_{t}, \delta_{t}$, and this prevents the squeezing of the components $u, \delta$.

Remark 2. We can express the constants in the proof of Theorem 2 and the radius of the absorbing set in terms of the parameters of the problem. Firstly, given $a_{i} \geq 0, i=1,2,3$, let us denote by $\gamma=\gamma\left(a_{1}, a_{2}, a_{3}\right)$ a nonnegative constant such that $a_{1} t^{4}+a_{2} t^{2}+a_{3} t \leq \gamma\left(t+t^{4}\right)$, for every $t \geq 0$. 
Hence, from (1.2) we have $|F(s)| \leq \widetilde{c}_{1}\left(|s|+s^{4}\right)$, for every $s \in \mathbb{R}$, where $\widetilde{c}_{1}=$ $\gamma\left(c_{1} / 2, c_{1} / 2,|f(0)|\right)$. Furthermore, from (1.7) we have $|G(s)| \leq \widetilde{c}_{2}\left(|s|+s^{2}\right)$, where $\widetilde{c}_{2}=\max \left\{c_{2} / 2,|g(0)|\right\}$. For the constants $\mu_{0}, c_{4}$ in (2.6) and $\mu_{1}, c_{5}$ in (2.7) we can assume (see [12, Remark 2])

$$
\mu_{0}=\min \left\{\left(1-\lambda_{0}\right) / 2,1\right\}, \quad c_{4}=|\Omega| C_{4},
$$

and

$$
\mu_{1}=\min \left\{\left(1-\lambda_{1}\right) / 2,1\right\}, \quad c_{5}=|\Gamma|_{2} C_{5},
$$

where

$$
\lambda_{0}=-\liminf _{|s| \rightarrow+\infty} f(s) / s \in \mathbb{R} \cup\{-\infty\}, \quad C_{4}=-2 \min _{|r| \leq \rho_{0}} F(r),
$$

with $\rho_{0} \geq 0$ such that $r f(r) \geq-\max \left\{\left(\lambda_{0}+1\right) / 2,0\right\} r^{2}$ for every $|r| \geq \rho_{0}$, and

$$
\lambda_{1}=-\liminf _{|s| \rightarrow+\infty} g(s) / s \in \mathbb{R} \cup\{-\infty\}, \quad C_{5}=-2 \min _{|r| \leq \rho_{1}} G(r),
$$

with $\rho_{1} \geq 0$ such that $r g(r) \geq-\max \left\{\left(\lambda_{1}+1\right) / 2,0\right\} r^{2}$ for every $|r| \geq \rho_{1}$. The constants $c_{6}, c_{7}$ in $(3.4),(3.5)$ can be given by

$$
c_{6}=|\Omega| \max \left\{C_{6}, 0\right\}, \quad c_{7}=|\Gamma|_{2} \max \left\{C_{7}, 0\right\},
$$

where $C_{6}=-\min _{|r| \leq \rho_{0}} r f(r)$ and $C_{7}=-\min _{|r| \leq \rho_{1}} r g(r)$. The constant $\alpha$ (see the definition of the function $J(\omega, \nu)$ in the proof of Theorem 2) is given by

$$
\alpha=\max \left\{\mu_{0}^{-1}, 1+4 c_{t}^{2} \mu_{0}^{-1},\left(2 \mu_{1}\right)^{-1}\right\},
$$

and for $\epsilon_{1}, \epsilon_{2}$ we can take the values $\epsilon_{1}=\min \left\{3 \mu^{*} /\left(1+c_{t}\right), 1 / 2\right\}, \epsilon_{2}=$ $\min \left\{4^{-1} c_{t}^{-2} \mu_{0}, 4^{-1} \mu_{1}\right\}$. Finally, $k_{1}, k_{2}$ in (3.10) can be expressed by

$$
k_{1}=\mu^{*}, \quad k_{2}=\gamma\left(2\left(\widetilde{c}_{1}|\Omega|^{1 / 2}+\widetilde{c}_{2}|\Gamma|_{2}^{1 / 2}\right), 8+2 \widetilde{c}_{2}+c_{t}^{2}, 2 \widetilde{c}_{1} c_{e}^{4}\right),
$$

where $c_{e}$ is the constant in the Sobolev embedding inequality $\|v\|_{L^{4}(\Omega)} \leq$ $c_{e}\|v\|_{1}$ for every $v \in H^{1}(\Omega)$ (we can assume $c_{e}=2 \sqrt{2}\|P\|, P: H^{1}(\Omega) \rightarrow$ $H^{1}\left(\mathbb{R}^{3}\right)$ being a linear bounded extension operator). Therefore, by means of (3.14) and (3.10), we can provide an estimate for the radius $R_{0}$ of the absorbing set

$$
R_{0}^{2}=\frac{k_{2}}{k_{1}}\left(2 \frac{c_{6}+c_{7}}{\mu^{*}}\right)^{1 / 2}\left(1+\left(2 \frac{c_{6}+c_{7}}{\mu^{*}}\right)^{3 / 2}\right)+\frac{c_{8}}{k_{1}} .
$$


Remark 3. (Uniform decay of the trajectories). A further application of Lemma 1 allows us to deduce a sufficient condition which ensures the decay in $\mathcal{H}$ of the trajectories, uniformly from every bounded subset $\mathcal{B} \subset \mathcal{H}$ (cf. [12, Remark 3]). Indeed, by applying Lemma 1 with $\eta>0$ left arbitrary (instead of fixing it equal to $\left.\left(c_{6}+c_{7}\right) J(\omega, \nu)\right)$, we deduce at once the desired sufficient condition, i.e. $c_{6}+c_{7}=c_{8}=0$, and, on account of Remark 2 as well, we can state the following

Proposition 1. Let (1.2), (1.3), (1.7), (1.8) hold. In addition, suppose that the following conditions

$$
\begin{aligned}
& \min _{|r| \leq \rho_{0}} r f(r) \geq 0, \quad \min _{|r| \leq \rho_{1}} r g(r) \geq 0, \\
& |\Omega| \min _{|r| \leq \rho_{0}} F(r)+|\Gamma|_{2} \min _{|r| \leq \rho_{1}} G(r)=0
\end{aligned}
$$

be satisfied, with $\rho_{0}, \rho_{1} \geq 0$ as in Remark 2. Then, for every $R>0$ we have

$$
\left\|S(t) w_{0}\right\|_{\mathcal{H}} \rightarrow 0 \quad \text { as } t \rightarrow+\infty
$$

uniformly for $\left\|w_{0}\right\|_{H} \leq R$. In particular, assumptions (3.16) hold if $s f(s) \geq 0$ and $s g(s) \geq 0$, for every $s \in \mathbb{R}$.

We conclude this section with an important corollary, concerning the uniform control of the dissipation integral, that we shall use in the following.

Corollary 2. For every $R>0$, there exists a positive constant $\Lambda=\Lambda(R)$ independent of $\omega, \nu$, such that, whenever $\left\|w_{0}\right\|_{\mathcal{H}} \leq R$, there holds

$$
\int_{0}^{\infty}\left(\omega\left\|u_{t}(\tau)\right\|^{2}+\nu\left\|\delta_{t}(\tau)\right\|_{L^{2}(\Gamma)}^{2}\right) d \tau \leq \Lambda
$$

\section{The global attractor}

In this section we deduce the existence of the global attractor $\mathcal{A}=\mathcal{A}_{\omega, \nu}$ for system (1.1). Our goal is to deduce the existence of $\mathcal{A}$ under the assumptions (1.2), (1.3) for the nonlinearity $f$. Unfortunately, the classical decomposition method, frequently used to prove asymptotic compactness in hyperbolic equations, seems problematic when only (1.2), (1.3) hold. Indeed this method usually requires stronger assumptions (e.g., like (1.4) and (1.5), see Section $5)$. The problem is due to the dynamic boundary conditions we have which prevent the possibility of using fractional power operators in order to obtain estimates in Sobolev spaces of higher order. 
The method we use to obtain the existence of $\mathcal{A}$ under the weaker assumptions (1.2), (1.3) relies on the use of an appropriate energy type equation. The idea to take advantage of energy equations to prove the existence of global attractors for weakly dissipative semigroup has been considered by J. M. Ball (see [1]), and has later been successfully employed by other authors in situations where the use of the decomposition method seems out of reach (see, for instance, [24], [1], and also [17], [30], [18], [23], [29], [19]).

Theorem 3. Let (1.2), (1.3) hold. Then, (1.1) possesses a unique global attractor $\mathcal{A}=\mathcal{A}_{\omega, \nu}$ in the energy phase space $\mathcal{H}$.

Theorem 3 is an immediate consequence of the existence of a bounded absorbing set, proved in Section 3, and of the asymptotic compactness of the semigroup, which we shall now prove by applying Ball's method.

In order to prove the asymptotic compactness, we first need to establish the following lemma, which concerns with the weak continuity property of the semigroup.

Lemma 4. The operators $S(t)$ are weakly continuous on $\mathcal{H}$, for all $t \geq 0$, i.e.

$$
w_{0 n} \rightarrow w_{0}, \quad \text { in } \mathcal{H} \Rightarrow S(t) w_{0 n} \rightarrow S(t) w_{0}, \quad \text { in } \mathcal{H} .
$$

Proof. The lemma is essentially proved in [1, Theorem 3.6]. Actually, the proof in [1, Theorem 3.6] applies to the solution of an abstract evolution equation in the form (2.2), with the assumptions (fulfilled in our case) that $A$ is a (generic) generator of a strongly continuous semigroup $e^{A t}$ of bounded linear operators on $\mathcal{H}$, and $\mathcal{F}$ is a sequentially weakly continuous map from $\mathcal{H}$ to $\mathcal{H}$.

We are now in a position to prove the asymptotic compactness of $S(t)$

Proposition 2. The semigroup $S(t)$ is asymptotically compact.

Proof. Let $w_{0 n}=\left(u_{0 n}, u_{1 n}, \delta_{0 n}, \delta_{1 n}\right)$ be a bounded sequence in $\mathcal{H}$ and $t_{n} \rightarrow \infty$. We have to show that the sequence

$$
w^{(n)}\left(t_{n}\right)=S\left(t_{n}\right) w_{0 n}=\left(u^{(n)}\left(t_{n}\right), u_{t}^{(n)}\left(t_{n}\right), \delta^{(n)}\left(t_{n}\right), \delta_{t}^{(n)}\left(t_{n}\right)\right)
$$

is precompact in $\mathcal{H}$.

Let us first consider the third and fourth components of the solution. By explicitly integrating the second equation in (1.1) written for the family of 
trajectories $w^{(n)}$, we obtain (it is not restrictive to consider the case $\nu=1$ )

$$
\begin{aligned}
& \delta^{(n)}\left(t_{n}\right)=\delta_{0 n} e^{-t_{n} / 2} \cos \left(\frac{\sqrt{3}}{2} t_{n}\right)+\frac{1}{\sqrt{3}}\left(\delta_{0 n}+2 \delta_{1 n}\right) e^{-t_{n} / 2} \sin \left(\frac{\sqrt{3}}{2} t_{n}\right) \\
& +k\left(t_{n}\right) u_{0 n}-\int_{0}^{t_{n}} k^{\prime}\left(t_{n}-\tau\right) u^{(n)}(\tau) d \tau
\end{aligned}
$$

where the kernel $k$ is given by $k(s)=(2 / \sqrt{3}) e^{-s / 2} \sin (s \sqrt{3} / 2)$.

Now, the first three terms on the right hand side of (4.1) obviously converge strongly to zero in $L^{2}(\Gamma)$. As far as the last term is concerned, we have

$$
\begin{aligned}
& \left\|\int_{0}^{t_{n}} k^{\prime}\left(t_{n}-\tau\right) u^{(n)}(\tau) d \tau\right\|_{1} \leq c \int_{0}^{t_{n}} e^{-\left(t_{n}-\tau\right) / 2}\left\|u^{(n)}(\tau)\right\|_{1} d \tau \\
& \leq 2 c M\left(1-e^{-t_{n} / 2}\right) \leq 2 c M
\end{aligned}
$$

for every $n \in \mathbb{N}$, where we have used the fact that $\left\|u^{(n)}(t)\right\|_{1} \leq M$, for all $t \geq 0$ and for all $n \in \mathbb{N}$, as a consequence of the existence of a bounded absorbing set $\mathcal{B}_{0}$, the constant $M$ depending on the radius $R_{0}$ of $\mathcal{B}_{0}$.

By means of the compactness of the trace operator from $H^{1}(\Omega)$ to $L^{2}(\Gamma)$, we thus deduce the precompactness in $L^{2}(\Gamma)$ of the last term on the right hand side of (4.1), which yields the precompactness in $L^{2}(\Gamma)$ of the sequence $\delta^{(n)}\left(t_{n}\right)$. The same argument obviously applies to the sequence $\delta_{t}^{(n)}\left(t_{n}\right)$ as well.

In order to prove the asymptotic compactness of the first two components of the solution, we introduce the following auxiliary functional

$$
\begin{aligned}
& I(w)=\frac{1}{2}\|u\|_{1}^{2}+\frac{1}{2}\left\|u_{t}\right\|^{2}+\frac{\omega}{2}\left(u, u_{t}\right) \\
& +\int_{\Omega} F(u)-\left\langle u, \delta_{t}\right\rangle-\frac{1}{2}\|u\|_{L^{2}(\Gamma)}^{2} .
\end{aligned}
$$

An easy calculation shows that $I(w(\cdot))$ satisfies

$$
\frac{d I}{d t}+\omega I+\frac{\omega}{2}\|u\|_{L^{2}(\Gamma)}^{2}=\left\langle u, \delta+\left(\nu-\frac{\omega}{2}\right) \delta_{t}\right\rangle+H(u),
$$

where

$$
H(u)=\omega \int_{\Omega}\left(F(u)-\frac{1}{2} f(u) u\right) .
$$

Now, since $\left\{S\left(t_{n}\right) w_{0 n}\right\}$ is bounded in $\mathcal{H}$ (due to the existence of a bounded absorbing set $\mathcal{B}_{0}$ ), we have, for a subsequence

$$
S\left(t_{n}\right) w_{0 n} \rightarrow w^{*}, \quad \text { in } \mathcal{H} .
$$


Similarly, for every $T \in \mathbb{N}$, the sequence $\left\{S\left(t_{n}-T\right) w_{0 n}\right\}$ has a weakly converging subsequence and, by means of a diagonalization procedure, we obtain a further subsequence (which we do not relabel) such that, for every $T \in \mathbb{N}$,

$$
S\left(t_{n}-T\right) w_{0 n} \rightarrow w_{T}, \quad \text { in } \mathcal{H},
$$

with $w_{T} \in \mathcal{B}_{0}$, for every $T \in \mathbb{N}$ (assuming that $\mathcal{B}_{0}$ is the closed ball given by (3.15)). By Lemma 4, from (4.6), (4.7) we get $S(T) w_{T}=w^{*}$.

We now multiply by $e^{\omega t}$ and integrate (4.4), written for each solution with initial data given by $S\left(t_{n}-T\right) w_{0 n}$. We get

$$
\begin{aligned}
& I\left(S\left(t_{n}\right) w_{0 n}\right)+\frac{\omega}{2} \int_{0}^{T} e^{-\omega(T-s)}\left\|\widetilde{u}^{(n)}(s)\right\|_{L^{2}(\Gamma)}^{2} d s=e^{-\omega T} I\left(S\left(t_{n}-T\right) w_{0 n}\right) \\
& +\int_{0}^{T} e^{-\omega(T-s)}\left\langle\widetilde{u}^{(n)}(s), \widetilde{\delta}^{(n)}(s)+\left(\nu-\frac{\omega}{2}\right) \widetilde{\delta}_{t}^{(n)}(s)\right\rangle d s \\
& +\int_{0}^{T} e^{-\omega(T-s)} H\left(\widetilde{u}^{(n)}(s)\right) d s
\end{aligned}
$$

where we have set

$$
\widetilde{w}^{(n)}(t)=\left(\widetilde{u}^{(n)}(t), \widetilde{u}_{t}^{(n)}(t), \widetilde{\delta}^{(n)}(t), \widetilde{\delta}_{t}^{(n)}(t)\right)=S\left(t+t_{n}-T\right) w_{0 n}
$$

Now, observe that, for every $t \geq 0$, we have $\widetilde{w}^{(n)}(t) \rightarrow S(t) w_{T}$ in $\mathcal{H}$, which implies $\widetilde{u}^{(n)}(t) \rightarrow \bar{u}(t)$ in $H^{1}(\Omega)$, where we have set $S(t) w_{T}=\left(\bar{u}(t), \bar{u}_{t}(t), \bar{\delta}(t), \bar{\delta}_{t}(t)\right)$. Hence

$$
\widetilde{u}^{(n)}(t) \rightarrow \bar{u}(t), \quad \text { strongly in } L^{2}(\Gamma),
$$

for every $t \geq 0$. This fact, together with the weak convergences

$$
\widetilde{\delta}^{(n)}(t) \rightarrow \bar{\delta}(t), \quad \widetilde{\delta}_{t}^{(n)}(t) \rightarrow \bar{\delta}_{t}(t), \quad \text { in } L^{2}(\Gamma),
$$

for every $t \geq 0$, allows to use the dominated convergence theorem in the second term on the right hand side of (4.8) to deduce that this term converges to

$$
\int_{0}^{T} e^{-\omega(T-s)}\left\langle\bar{u}(s), \bar{\delta}(s)+\left(\nu-\frac{\omega}{2}\right) \bar{\delta}_{t}(s)\right\rangle d s .
$$

Similarly, the second term on the left hand side of (4.8) converges to

$$
\frac{\omega}{2} \int_{0}^{T} e^{-\omega(T-s)}\|\bar{u}(s)\|_{L^{2}(\Gamma)}^{2} d s,
$$

while the last term on the right hand side converges to

$$
\int_{0}^{T} e^{-\omega(T-s)} H(\bar{u}(s)) d s
$$


by virtue of the sequential weak continuity of the map $H: H^{1}(\Omega) \rightarrow \mathbb{R}$, which can be easily proved by using (1.2) (see [1, Theorem 3.6]).

Hence, from (4.8) we obtain

$$
\begin{aligned}
& \limsup _{n \rightarrow \infty} I\left(S\left(t_{n}\right) w_{0 n}\right)+\frac{\omega}{2} \int_{0}^{T} e^{-\omega(T-s)}\|\bar{u}(s)\|_{L^{2}(\Gamma)}^{2} d s \\
& \leq C e^{-\omega T}+\int_{0}^{T} e^{-\omega(T-s)}\left\langle\bar{u}(s), \bar{\delta}(s)+\left(\nu-\frac{\omega}{2}\right) \bar{\delta}_{t}(s)\right\rangle d s \\
& +\int_{0}^{T} e^{-\omega(T-s)} H(\bar{u}(s)) d s
\end{aligned}
$$

for every $T \in \mathbb{N}$. On the other hand, by integrating (4.4) written for the solution with initial data $w_{T}$, we have

$$
\begin{aligned}
& I\left(w^{*}\right)+\frac{\omega}{2} \int_{0}^{T} e^{-\omega(T-s)}\|\bar{u}(s)\|_{L^{2}(\Gamma)}^{2} d s \\
& =e^{-\omega T} I\left(w_{T}\right)+\int_{0}^{T} e^{-\omega(T-s)}\left\langle\bar{u}(s), \bar{\delta}(s)+\left(\nu-\frac{\omega}{2}\right) \bar{\delta}_{t}(s)\right\rangle d s \\
& +\int_{0}^{T} e^{-\omega(T-s)} H(\bar{u}(s)) d s,
\end{aligned}
$$

for every $T \in \mathbb{N}$. From (4.9) and (4.10) we find

$$
\limsup _{n \rightarrow \infty} I\left(S\left(t_{n}\right) w_{0 n}\right) \leq C e^{-\omega T}+I\left(w^{*}\right),
$$

and by letting $T \rightarrow \infty$, we see that $\limsup _{n \rightarrow \infty} I\left(S\left(t_{n}\right) w_{0 n}\right) \leq I\left(w^{*}\right)$. But, by the weak lower semicontinuity of the norm, it is easy to see that, up to a subsequence, we have $\liminf _{n \rightarrow \infty} I\left(S\left(t_{n}\right) w_{o n}\right) \geq I\left(w^{*}\right)$. Therefore $I\left(S\left(t_{n}\right) w_{0 n}\right) \rightarrow$ $I\left(w^{*}\right)$, and hence

$$
\left\|u^{(n)}\left(t_{n}\right)\right\|_{1}^{2}+\left\|u_{t}^{(n)}\left(t_{n}\right)\right\|^{2} \rightarrow\left\|w_{1}^{*}\right\|_{1}^{2}+\left\|w_{2}^{*}\right\|^{2},
$$

where $w^{*}=\left(w_{1}^{*}, w_{2}^{*}, w_{3}^{*}, w_{4}^{*}\right)$. This fact, together with the weak convergence (4.6), yields $u^{(n)}\left(t_{n}\right) \rightarrow w_{1}^{*}$ in $H^{1}(\Omega)$ strongly and $u_{t}^{(n)}\left(t_{n}\right) \rightarrow w_{2}^{*}$ in $L^{2}(\Omega)$ strongly. Thus, the first two components of the solution as well are asymptotically compact, and the proof is now complete.

\section{$5 \quad$ Regular attracting sets}

In order to prove the existence of a regular attracting set, which provides the regularity of the attractor and will also be useful for the construction of 
an exponential attractor, we shall use a quite effective decomposition of the solution, that has already been exploited in [28] and in other papers (see [16], [20] and [32]).

This approach is particularly suitable for our problem, where fractional operators cannot be used, and requires, in addition, some more restrictive assumptions on the nonlinearity, i.e., (1.4) and (1.5).

In the following $c \geq 0$ will stand for a generic constant, that may vary even within the same equation, depending on the radius $R_{0}$ of the absorbing set $\mathcal{B}_{0}$, but independent of $\omega, \nu$. Moreover $c_{\omega, \nu}$ and $b_{\omega, \nu}$ will stand for some constants depending on $R_{0}, \omega$ and $\nu$.

Theorem 4. Let (1.2)-(1.5) hold. Then, there exists a subset $\mathcal{B}_{1}=\mathcal{B}_{1}(\omega, \nu) \subset$ $\mathcal{H}_{1}$ closed and bounded in $\mathcal{H}_{1}$ such that

$$
\operatorname{dist}_{\mathcal{H}}\left(S(t) \mathcal{B}_{0}, \mathcal{B}_{1}\right) \leq c_{\omega, \nu} e^{-c J(\omega, \nu) t}
$$

for every $t \geq 0$.

By virtue of the minimality property of the global attractor, we immediately have the following.

Corollary 3. Under the assumptions (1.2)-(1.5), the global attractor $\mathcal{A}$ of the semigroup on $\mathcal{H}$ associated with (1.1) is contained and bounded in $\mathcal{H}_{1}$.

In order to prove Theorem 4 , we consider the initial data $w_{0} \in \mathcal{B}_{0}$ and we decompose the solution $w$ into the sum $w=w^{d}+w^{c}$, where $w^{d}=$ $\left(u^{d}, u_{t}^{d}, \delta^{d}, \delta_{t}^{d}\right)$ and $w^{c}=\left(u^{c}, u_{t}^{c}, \delta^{c}, \delta_{t}^{c}\right)$ are the solutions to the following problems;

$$
\left\{\begin{array}{l}
u_{t t}^{d}+\omega u_{t}^{d}-\Delta u^{d}+u^{d}+\psi(u)-\psi\left(u^{c}\right)=0 \quad \text { in } \Omega \times(0, \infty) \\
\delta_{t t}^{d}+\nu \delta_{t}^{d}+\delta^{d}=-u_{t}^{d} \quad \text { on } \Gamma \times(0, \infty) \\
\delta_{t}^{d}=\frac{\partial u^{d}}{\partial \mathbf{n}} \quad \text { on } \Gamma \times(0, \infty) \\
w^{d}(0)=w_{0} \quad \text { in } \Omega
\end{array}\right.
$$

and

$$
\left\{\begin{array}{l}
u_{t t}^{c}+\omega u_{t}^{c}-\Delta u^{c}+u^{c}+\psi\left(u^{c}\right)=\theta u \quad \text { in } \Omega \times(0, \infty) \\
\delta_{t t}^{c}+\nu \delta_{t}^{c}+\delta^{c}=-u_{t}^{c} \quad \text { on } \Gamma \times(0, \infty) \\
\delta_{t}^{c}=\frac{\partial u^{c}}{\partial \mathbf{n}} \quad \text { on } \Gamma \times(0, \infty) \\
w^{c}(0)=0 \quad \text { in } \Omega ;
\end{array}\right.
$$

here we have set

$$
\psi(s):=f(s)+\theta s
$$

with $\theta \geq l$ in order to have $\psi^{\prime}(s) \geq 0$, by (1.5).

In the next lemmas we denote by $k=k_{\omega, \nu}$ a positive constant depending on $\omega, \nu$, and $R_{0}$, having the form $k_{\omega, \nu}=c /(\omega J(\omega, \nu))$. 
Lemma 5. We have

$$
\left\|w^{c}(t)\right\|_{\mathcal{H}} \leq k_{\omega, \nu}
$$

for every $t \geq 0$ and every $w_{0} \in \mathcal{B}_{0}$.

Proof. We can argue as in the proof of Theorem 2. Indeed, we can rewrite (3.1) for system (5.3), replacing $F(u)$ with $\Psi\left(u^{c}\right):=\int_{0}^{u^{c}} \psi(s) d s$ and adding on the right hand side the additional term $\theta\left(u, \xi^{c}\right) \leq \frac{\omega}{2}\left\|\xi^{c}\right\|^{2}+\frac{c}{\omega}\|u\|_{1}^{2} \leq$ $\frac{\omega}{2}\left\|\xi^{c}\right\|^{2}+\frac{c}{\omega}$, by (2.8). We are finally led to

$$
\frac{d}{d t} E\left(w^{c}(t)\right)+c J(\omega, \nu)\left\|w^{c}(t)\right\|_{\mathcal{H}}^{2} \leq c J(\omega, \nu)+\frac{c}{\omega} .
$$

Now, by means of Lemma 1 , we easily get $\left\|w^{c}(t)\right\|_{\mathcal{H}} \leq c /(\omega J(\omega, \nu))$, for all $t \geq t_{0}:=c / J(\omega, \nu)$. On the other hand, by multiplying in $L^{2}(\Omega)$ the first equation in $(5.3)$ by $u_{t}^{c}$, in $L^{2}(\Gamma)$ the second by $\delta_{t}^{c}$ and adding the resulting equations together, we have

$$
\begin{aligned}
& \frac{1}{2} \frac{d}{d t}\left\{\left\|w^{c}(t)\right\|_{\mathcal{H}}^{2}+2 \int_{\Omega} \Psi\left(u^{c}\right)\right\}+\omega\left\|u_{t}^{c}\right\|^{2}+\nu\left\|\delta_{t}^{c}\right\|_{L^{2}(\Gamma)}^{2} \\
& =\theta\left(u, u_{t}^{c}\right) \leq \frac{\omega}{2}\left\|u_{t}^{c}\right\|^{2}+\frac{c}{\omega} .
\end{aligned}
$$

Integrating (5.5) between 0 and $t \in\left[0, t_{0}\right]$, we are led to $\left\|w^{c}(t)\right\|_{\mathcal{H}} \leq c \sqrt{t_{0} / \omega}=$ $c / \sqrt{\omega J(\omega, \nu)}$, for all $t \in\left[0, t_{0}\right]$. We thus get (5.4), with the constant $k_{\omega, \nu}$ in the form written above.

Lemma 6. For every $\epsilon>0$ there exists $m_{\epsilon}>0$, depending also on $\omega$ and $\nu$, such that, for every $0 \leq s \leq t$ and every $w_{0} \in \mathcal{B}_{0}$, we have

$$
\int_{s}^{t}\left(\left\|u_{t}(\tau)\right\|^{2}+\left\|u_{t}^{c}(\tau)\right\|^{2}\right) d \tau \leq \frac{\epsilon}{2}(t-s)+m_{\epsilon}
$$

Proof. From (5.5), on account of Lemma 5, we can write

$$
\begin{aligned}
& \frac{1}{2} \frac{d}{d t}\left\{\left\|w^{c}(t)\right\|_{\mathcal{H}}^{2}+2 \int_{\Omega} \Psi\left(u^{c}\right)-2 \theta\left(u, u^{c}\right)\right\}+\omega\left\|u_{t}^{c}\right\|^{2}+\nu\left\|\delta_{t}^{c}\right\|_{L^{2}(\Gamma)}^{2} \\
& =-\theta\left(u_{t}, u^{c}\right) \leq \omega \frac{\epsilon}{2}+\frac{c k^{2}}{\omega \epsilon}\left\|u_{t}\right\|^{2} .
\end{aligned}
$$

Setting

$$
\Lambda:=\left\|w^{c}\right\|_{\mathcal{H}}^{2}+2 \int_{\Omega} \Psi\left(u^{c}\right)-2 \theta\left(u, u^{c}\right)
$$

we easily see that $|\Lambda(t)| \leq c k^{4}$, for all $t \geq 0$, by Lemma 5, (2.8) and (1.2). Now, by integrating (5.7) between $s$ and $t$ and by using Corollary 2, we get (5.6). The constant $m_{\epsilon}$ can be expressed, as a function of $\omega$ and $\nu$, by $m_{\epsilon}=c k^{2} /\left(\epsilon \omega^{3}\right)+c k^{4} / \omega$. 
We now prove

Lemma 7. We have

$$
\left\|w^{d}(t)\right\|_{\mathcal{H}} \leq c_{\omega, \nu} e^{-c J(\omega, \nu) t}
$$

for every $t \geq 0$ and every $w_{0} \in \mathcal{B}_{0}$.

Proof. We multiply $(5.2)_{1}$ in $L^{2}(\Omega)$ by $\xi^{d}:=u_{t}^{d}+\epsilon u^{d}$ and $(5.2)_{2}$ in $L^{2}(\Gamma)$ by $\zeta^{d}:=\delta_{t}^{d}+\epsilon \delta^{d}$, where $\epsilon>0$ shall be fixed later. By also taking into account $(5.2)_{3}$, we find the following equality

$$
\begin{aligned}
& \frac{1}{2} \frac{d}{d t}\left\{\left\|u^{d}\right\|_{1}^{2}+\left\|\xi^{d}\right\|^{2}+\left\|\delta^{d}\right\|_{L^{2}(\Gamma)}^{2}+\left\|\zeta^{d}\right\|_{L^{2}(\Gamma)}^{2}+2 \epsilon\left\langle u^{d}, \delta^{d}\right\rangle\right. \\
& \left.+2\left(\psi(u)-\psi\left(u^{c}\right), u^{d}\right)-\left(\psi^{\prime}(u) u^{d}, u^{d}\right)\right\}+\epsilon\left\|u^{d}\right\|_{1}^{2}+(\omega-\epsilon)\left\|\xi^{d}\right\|^{2} \\
& -\epsilon(\omega-\epsilon)\left(u^{d}, \xi^{d}\right)+\epsilon\left\|\delta^{d}\right\|_{L^{2}(\Gamma)}^{2}+(\nu-\epsilon)\left\|\zeta^{d}\right\|_{L^{2}(\Gamma)}^{2}-\epsilon(\nu-\epsilon)\left\langle\delta^{d}, \zeta^{d}\right\rangle \\
& +\epsilon\left(\psi(u)-\psi\left(u^{c}\right), u^{d}\right)=2 \epsilon\left\langle u^{d}, \zeta^{d}\right\rangle-2 \epsilon^{2}\left\langle u^{d}, \delta^{d}\right\rangle \\
& +\left(\left(\psi^{\prime}(u)-\psi^{\prime}\left(u^{c}\right)\right) u_{t}^{c}, u^{d}\right)-\frac{1}{2}\left(\psi^{\prime \prime}(u) u_{t},\left(u^{d}\right)^{2}\right) .
\end{aligned}
$$

We now have

$$
\begin{aligned}
& \left|\left(\psi^{\prime}(u) u^{d}, u^{d}\right)\right| \leq c\left(1+\|u\|_{1}^{2}\right)\left\|u^{d}\right\|_{1}\left\|u^{d}\right\| \\
& \leq c\left\|u^{d}\right\|_{1}\left\|u^{d}\right\| \leq \frac{1}{2}\left\|u^{d}\right\|_{1}^{2}+c\left\|u^{d}\right\|^{2}
\end{aligned}
$$

and hence, by means of assumption (1.5), we have

$$
\begin{aligned}
& 2\left(\psi(u)-\psi\left(u^{c}\right), u^{d}\right)-\left(\psi^{\prime}(u) u^{d}, u^{d}\right) \geq 2(\theta-l)\left\|u^{d}\right\|^{2}-\frac{1}{2}\left\|u^{d}\right\|_{1}^{2}-c\left\|u^{d}\right\|^{2} \\
& \geq-\frac{1}{2}\left\|u^{d}\right\|_{1}^{2}
\end{aligned}
$$

provided $\theta$ is chosen large enough $(\theta \geq l+c / 2)$. We now set (we omit the indication of the time $t$ on the right)

$$
\begin{aligned}
& \Phi(t):=\left\|u^{d}\right\|_{1}^{2}+\left\|\xi^{d}\right\|^{2}+\left\|\delta^{d}\right\|_{L^{2}(\Gamma)}^{2}+\left\|\zeta^{d}\right\|_{L^{2}(\Gamma)}^{2}+2 \epsilon\left\langle u^{d}, \delta^{d}\right\rangle \\
& +2\left(\psi(u)-\psi\left(u^{c}\right), u^{d}\right)-\left(\psi^{\prime}(u) u^{d}, u^{d}\right)
\end{aligned}
$$

and, on account of (5.10) and of the trace inequality $\left\|u^{d}\right\|_{L^{2}(\Gamma)} \leq c\left\|u^{d}\right\|_{1}$, it is easy to check that, provided $\epsilon \in\left(0, \epsilon_{1}^{\prime}\right]$, where $\epsilon_{1}^{\prime}$ is small enough and independent of $\omega, \nu$, we have

$$
\Phi(t) \geq \frac{1}{4}\left\|w^{d}(t)\right\|_{\mathcal{H}}^{2}
$$


for every $t \geq 0$ and every $w_{0} \in \mathcal{B}_{0}$. On the other hand, by using Lemma 5 , we have

$$
2\left|\left(\psi(u)-\psi\left(u^{c}\right), u^{d}\right)\right| \leq c\left(1+\|u\|_{1}^{2}+\left\|u^{c}\right\|_{1}^{2}\right)\left\|u^{d}\right\|_{1}^{2} \leq c k^{2}\left\|u^{d}\right\|_{1}^{2},
$$

where the constant $k=k_{\omega, \nu}$ is the same as in Lemma 5. Furthermore

$$
\left|\left(\psi^{\prime}(u) u^{d}, u^{d}\right)\right| \leq c\left(1+\|u\|_{1}^{2}\right)\left\|u^{d}\right\|_{1}^{2} \leq c\left\|u^{d}\right\|_{1}^{2} .
$$

Hence, it is also easy to check that

$$
\Phi(t) \leq c k^{2}\left\|w^{d}(t)\right\|_{\mathcal{H}}^{2},
$$

for every $t \geq 0$ and every $w_{0} \in \mathcal{B}_{0}$. Let us now rewrite (5.9) in terms of the functional $\Phi$,

$$
\begin{aligned}
& \frac{d \Phi}{d t}+\epsilon \Phi+\epsilon\left\|u^{d}\right\|_{1}^{2}+(2 \omega-3 \epsilon)\left\|\xi^{d}\right\|^{2} \\
& +\epsilon\left\|\delta^{d}\right\|_{L^{2}(\Gamma)}^{2}+(2 \nu-3 \epsilon)\left\|\zeta^{d}\right\|_{L^{2}(\Gamma)}^{2}-2 \epsilon(\omega-\epsilon)\left(u^{d}, \xi^{d}\right) \\
& -2 \epsilon(\nu-\epsilon)\left\langle\delta^{d}, \zeta^{d}\right\rangle-2 \epsilon^{2}\left\langle u^{d}, \delta^{d}\right\rangle+\epsilon\left(\psi^{\prime}(u) u^{d}, u^{d}\right) \\
& =4 \epsilon\left\langle u^{d}, \zeta^{d}\right\rangle-4 \epsilon^{2}\left\langle u^{d}, \delta^{d}\right\rangle+2\left(\left(\psi^{\prime}(u)-\psi^{\prime}\left(u^{c}\right)\right) u_{t}^{c}, u^{d}\right) \\
& -\left(\psi^{\prime \prime}(u) u_{t},\left(u^{d}\right)^{2}\right) .
\end{aligned}
$$

We now have

$$
\begin{aligned}
& -2 \epsilon(\omega-\epsilon)\left(u^{d}, \xi^{d}\right) \geq-\frac{\epsilon}{8}\left\|u^{d}\right\|_{1}^{2}-\epsilon \omega^{2} c\left\|\xi^{d}\right\|^{2}, \\
& -2 \epsilon(\nu-\epsilon)\left\langle\delta^{d}, \zeta^{d}\right\rangle \geq-\frac{\epsilon}{2}\left\|\delta^{d}\right\|_{L^{2}(\Gamma)}^{2}-2 \epsilon \nu^{2}\left\|\zeta^{d}\right\|_{L^{2}(\Gamma)}^{2}, \\
& -2 \epsilon^{2}\left\langle u^{d}, \delta^{d}\right\rangle \leq-\frac{\epsilon}{8}\left\|u^{d}\right\|_{1}^{2}-c \epsilon^{3}\left\|\delta^{d}\right\|_{L^{2}(\Gamma)}^{2}, \\
& 4 \epsilon\left\langle u^{d}, \zeta^{d}\right\rangle \leq \frac{\epsilon}{8}\left\|u^{d}\right\|_{1}^{2}+c \epsilon\left\|\zeta^{d}\right\|_{L^{2}(\Gamma)}^{2}, \\
& -4 \epsilon^{2}\left\langle u^{d}, \delta^{d}\right\rangle \leq \frac{\epsilon}{8}\left\|u^{d}\right\|_{1}^{2}+c \epsilon^{3}\left\|\delta^{d}\right\|_{L^{2}(\Gamma)}^{2} .
\end{aligned}
$$

Furthermore, by means of (5.12) we have (assuming $\epsilon \leq \epsilon_{1}^{\prime}$ )

$$
\begin{aligned}
& 2\left|\left(\left(\psi^{\prime}(u)-\psi^{\prime}\left(u^{c}\right)\right) u_{t}^{c}, u^{d}\right)\right| \leq c\left(1+\|u\|_{1}+\left\|u^{c}\right\|_{1}\right)\left\|u_{t}^{c}\right\|\left\|u^{d}\right\|_{1}^{2} \\
& \leq c k\left\|u_{t}^{c}\right\|\left\|u^{d}\right\|_{1}^{2} \leq \frac{\epsilon}{8}\left\|u^{d}\right\|_{1}^{2}+\frac{c k^{2}}{\epsilon}\left\|u_{t}^{c}\right\|^{2}\left\|u^{d}\right\|_{1}^{2} \\
& \leq \frac{\epsilon}{8}\left\|u^{d}\right\|_{1}^{2}+\frac{c k^{2}}{\epsilon}\left\|u_{t}^{c}\right\|^{2} \Phi,
\end{aligned}
$$


and also

$$
\begin{aligned}
& \left|\left(\psi^{\prime \prime}(u) u_{t},\left(u^{d}\right)^{2}\right)\right| \leq c\left(1+\|u\|_{1}\right)\left\|u^{d}\right\|_{1}^{2}\left\|u_{t}\right\| \\
& \leq c\left\|u_{t}\right\|\left\|u^{d}\right\|_{1}^{2} \leq \frac{\epsilon}{8}\left\|u^{d}\right\|_{1}^{2}+\frac{c}{\epsilon}\left\|u_{t}\right\|^{2}\left\|u^{d}\right\|_{1}^{2} \\
& \leq \frac{\epsilon}{8}\left\|u^{d}\right\|^{2}+\frac{c}{\epsilon}\left\|u_{t}\right\|^{2} \Phi .
\end{aligned}
$$

Hence, plugging (5.15)-(5.21) into (5.14) and choosing $\epsilon \leq \min \left\{\epsilon_{1}^{\prime}, \epsilon_{2}^{\prime}, K(\omega)\right.$, $K(\nu)\}=c J(\omega, \nu)$, where $\epsilon_{2}^{\prime}$ is independent of $\omega$ and $\nu$, we get

$$
\begin{aligned}
& \frac{d \Phi}{d t}+\epsilon \Phi+\frac{\epsilon}{4}\left\|u^{d}\right\|_{1}^{2}+\omega\left\|\xi^{d}\right\|^{2}+\frac{\epsilon}{4}\left\|\delta^{d}\right\|_{L^{2}(\Gamma)}^{2}+\nu\left\|\zeta^{d}\right\|_{L^{2}(\Gamma)}^{2} \\
& \leq \frac{c k^{2}}{\epsilon}\left(\left\|u_{t}^{c}\right\|^{2}+\left\|u_{t}\right\|^{2}\right) \Phi .
\end{aligned}
$$

Now, choosing $\epsilon=c J(\omega, \nu)$ in (5.22), by means of Lemma 6, Lemma 2 and (5.12), (5.13), we finally get (5.8) with $c_{\omega, \nu}$ having the form $c_{\omega, \nu}=$ $c k_{\omega, \nu} \exp \left\{c k_{\omega, \nu}^{6} /\left(\omega^{3} J^{3}(\omega, \nu)\right)\right\}$.

Lemma 8. We have

$$
\left\|w^{c}(t)\right\|_{\mathcal{H}_{1}} \leq b_{\omega, \nu}
$$

for every $t \geq 0$ and every $w_{0} \in \mathcal{B}_{0}$.

Proof. We differentiate $(5.3)_{1}$ with respect to time and, setting $v^{c}:=u_{t}^{c}$, we obtain

$$
v_{t t}^{c}+\omega v_{t}^{c}-\Delta v^{c}+v^{c}+\psi^{\prime}\left(u^{c}\right) v^{c}=\theta u_{t} .
$$

Then, we multiply (5.23) in $L^{2}(\Omega)$ by $v_{t}^{c}+\epsilon v^{c}$, where $\epsilon>0$ is to be determined later. By taking into account $(5.3)_{2}$ and $(5.3)_{3}$, after some calculations we are led to the following differential identity

$$
\begin{aligned}
& \frac{d}{d t}\left\{\left\|v^{c}\right\|_{1}^{2}+\left\|v_{t}^{c}\right\|^{2}+\left\|v^{c}\right\|_{L^{2}(\Gamma)}^{2}+\epsilon \omega\left\|v^{c}\right\|^{2}+2\left\langle\nu \delta_{t}^{c}+\delta^{c}, v^{c}\right\rangle\right. \\
& \left.+2 \epsilon\left(v^{c}, v_{t}^{c}\right)+\left(\psi^{\prime}\left(u^{c}\right) v^{c}, v^{c}\right)\right\}+2 \epsilon\left\|v^{c}\right\|_{1}^{2}+2(\omega-\epsilon)\left\|v_{t}^{c}\right\|^{2} \\
& +2(\epsilon+\nu)\left\|v^{c}\right\|_{L^{2}(\Gamma)}^{2}+2 \epsilon\left\langle\nu \delta_{t}^{c}+\delta^{c}, v^{c}\right\rangle+2\left(\nu^{2}-1\right)\left\langle\delta_{t}^{c}, v^{c}\right\rangle \\
& +2 \nu\left\langle\delta^{c}, v^{c}\right\rangle+2 \epsilon\left(\psi^{\prime}\left(u^{c}\right) v^{c}, v^{c}\right)=\left(\psi^{\prime \prime}\left(u^{c}\right) u_{t}^{c},\left(v^{c}\right)^{2}\right) \\
& +2 \theta\left(u_{t}, v_{t}^{c}\right)+2 \epsilon \theta\left(u_{t}, u_{t}^{c}\right)
\end{aligned}
$$

We now introduce the functional

$$
\begin{aligned}
& \Lambda_{1}:=\left\|v^{c}\right\|_{1}^{2}+\left\|v_{t}^{c}\right\|^{2}+\left\|v^{c}\right\|_{L^{2}(\Gamma)}^{2}+\epsilon \omega\left\|v^{c}\right\|^{2}+2\left\langle\nu \delta_{t}^{c}+\delta^{c}, v^{c}\right\rangle \\
& +2 \epsilon\left(v^{c}, v_{t}^{c}\right)+\left(\psi^{\prime}\left(u^{c}\right) v^{c}, v^{c}\right)
\end{aligned}
$$


and observe that, by choosing $\epsilon \in\left(0, \epsilon_{3}^{\prime}\right]$, with $\epsilon_{3}^{\prime}$ small enough and independent of $\omega$ and $\nu$, we have

$$
\Lambda_{1} \geq \frac{1}{2}\left(\left\|v^{c}\right\|_{1}^{2}+\left\|v_{t}^{c}\right\|^{2}+\left\|v^{c}\right\|_{L^{2}(\Gamma)}^{2}\right)-2(\nu+1)^{2} k^{2} .
$$

Furthermore, by means of (1.2) we also have

$\Lambda_{1} \leq\left(1+c k^{2}+\epsilon c\right)\left\|v^{c}\right\|_{1}^{2}+(1+\epsilon c)\left\|v_{t}^{c}\right\|^{2}+(1+\epsilon \omega)\left\|v^{c}\right\|_{L^{2}(\Gamma)}^{2}+2(\nu+1) k\left\|v^{c}\right\|_{L^{2}(\Gamma)}$.

In terms of functional $\Lambda_{1}$, the differential identity (5.24) can be written in the form

$$
\begin{aligned}
& \frac{d \Lambda_{1}}{d t}+\epsilon \Lambda_{1}+\epsilon\left\|v^{c}\right\|_{1}^{2}+(2 \omega-3 \epsilon)\left\|v_{t}^{c}\right\|^{2}+(2 \nu+\epsilon)\left\|v^{c}\right\|_{L^{2}(\Gamma)}^{2} \\
& -\omega \epsilon^{2}\left\|v^{c}\right\|^{2}-2 \epsilon^{2}\left(v^{c}, v_{t}^{c}\right)+2\left(\nu^{2}-1\right)\left\langle v^{c}, \delta_{t}^{c}\right\rangle+2 \nu\left\langle v^{c}, \delta^{c}\right\rangle \\
& +\epsilon\left(\psi^{\prime}\left(u^{c}\right) v^{c}, v^{c}\right)=\left(\psi^{\prime \prime}\left(u^{c}\right) u_{t}^{c},\left(v^{c}\right)^{2}\right) \\
& +2 \theta\left(u_{t}, v_{t}^{c}\right)+2 \epsilon \theta\left(u_{t}, u_{t}^{c}\right)
\end{aligned}
$$

We now have

$$
\begin{gathered}
-2 \epsilon^{2}\left(v^{c}, v_{t}^{c}\right) \geq-\epsilon^{2} \omega\left\|v^{c}\right\|_{1}^{2}-\frac{\epsilon^{2}}{\omega}\left\|v_{t}^{c}\right\|^{2}, \\
2 \nu\left\langle v^{c}, \delta^{c}\right\rangle \geq-\frac{\epsilon}{2}\left\|v^{c}\right\|_{L^{2}(\Gamma)}^{2}-\frac{2 \nu^{2} k^{2}}{\epsilon}, \\
2\left(\nu^{2}-1\right)\left\langle v^{c}, \delta_{t}^{c}\right\rangle \geq-\frac{\epsilon}{2}\left\|v^{c}\right\|_{L^{2}(\Gamma)}^{2}-\frac{2\left(\nu^{2}-1\right)^{2} k^{2}}{\epsilon},
\end{gathered}
$$

where we have exploited the control $\left\|\delta^{c}(t)\right\|_{L^{2}(\Gamma)} \leq k$ (by Lemma 5). Furthermore

$$
\begin{aligned}
& 2 \theta\left(u_{t}, v_{t}^{c}\right)+2 \epsilon \theta\left(u_{t}, u_{t}^{c}\right) \leq c\left\|v_{t}^{c}\right\|+\epsilon c k \\
& \leq \omega\left\|v_{t}^{c}\right\|^{2}+\frac{c}{\omega}+\epsilon c k
\end{aligned}
$$

and, by (5.26)

$$
\begin{aligned}
& \left(\psi^{\prime \prime}\left(u^{c}\right) u_{t}^{c},\left(v^{c}\right)^{2}\right) \leq c\left(1+\left\|u^{c}\right\|_{1}\right)\left\|u_{t}^{c}\right\|\left\|v^{c}\right\|_{1}^{2} \\
& \leq c k\left\|u_{t}^{c}\right\|\left\|v^{c}\right\|_{1}^{2} \leq c k\left\|u_{t}^{c}\right\| \Lambda_{1}+c k^{3}(\nu+1)^{2}\left\|u_{t}^{c}\right\| .
\end{aligned}
$$

Plugging (5.29)- (5.33) into (5.28) and by taking $\epsilon$ small enough (i.e. $0<\epsilon \leq$ $\left.\min \left\{\epsilon_{3}^{\prime}, c / \omega, c \omega\right\}\right)$, we get the following differential inequality

$$
\frac{d \Lambda_{1}}{d t}+\epsilon \Lambda_{1} \leq c k\left\|u_{t}^{c}\right\| \Lambda_{1}+c k^{3}(\nu+1)^{2}\left\|u_{t}^{c}\right\|+b_{1}
$$


where the constant $b_{1}$ has the form $b_{1}=2\left(\nu^{2}-1\right)^{2} k^{2} / \epsilon+2 \nu^{2} k^{2} / \epsilon+c / \omega+\epsilon c k$. Henceforth in this proof we denote by $b_{i}$ a nonnegative constant depending on $R_{0}, \omega$ and $\nu$. We now have, by means of Corollary 2 and Lemma 7

$$
\int_{0}^{+\infty}\left\|u_{t}^{c}(\tau)\right\|^{2} d \tau \leq 2 \int_{0}^{+\infty}\left(\left\|u_{t}(\tau)\right\|^{2}+\left\|u_{t}^{d}(\tau)\right\|^{2}\right) d \tau \leq b_{2}
$$

where $b_{2}=c\left(\omega^{-1}+c_{\omega, \nu}^{2} J(\omega, \nu)^{-1}\right)$. Therefore

$$
c k \int_{s}^{t}\left\|u_{t}^{c}(\tau)\right\| d \tau \leq b_{3}(t-s)^{1 / 2}
$$

with $b_{3}=c k b_{2}^{1 / 2}$. Since we also have

$$
\int_{t}^{t+1}\left(c k^{3}(\nu+1)^{2}\left\|u_{t}^{c}\right\|+b_{1}\right) d \tau \leq b_{4}
$$

with $b_{4}=c k^{3}(\nu+1)^{2} b_{2}^{1 / 2}+b_{1}$, we are thus in the hypotheses of Lemma 3 and so we conclude that

$$
\Lambda_{1}(t) \leq \gamma \Lambda_{1}(0) e^{-\epsilon t / 2}+K,
$$

for all $t \geq 0$. The constants $\gamma$ and $K$ can be expressed in terms of $\omega, \nu$ (see Lemma 3). On the other hand, from (5.27) and (5.3) $)_{1}, \Lambda_{1}(0) \leq b_{5}$ and from (5.26) we get the bound

$$
\left\|u_{t}^{c}(t)\right\|_{1}+\left\|u_{t t}^{c}(t)\right\| \leq b_{6} .
$$

Equation $(5.3)_{1}$ finally yields the control $\left\|\Delta u^{c}(t)\right\| \leq b_{7}$.

We now multiply equation $(5.3)_{2}$ by $\left(-\Delta_{\Gamma}\right)^{1 / 2}\left(\delta_{t}^{c}+\epsilon \delta^{c}\right)$ in $L^{2}(\Gamma)$, where $\epsilon>$ 0 is to be determined later. After some calculations we obtain the following identity

$$
\begin{aligned}
& \frac{1}{2} \frac{d}{d t}\left\{(1+\epsilon \nu)\left\|\left(-\Delta_{\Gamma}\right)^{1 / 4} \delta^{c}\right\|_{L^{2}(\Gamma)}^{2}+\left\|\left(-\Delta_{\Gamma}\right)^{1 / 4} \delta_{t}^{c}\right\|_{L^{2}(\Gamma)}^{2}+\left\|\left(-\Delta_{\Gamma}\right)^{1 / 4} u^{c}\right\|_{L^{2}(\Gamma)}^{2}\right. \\
& +2 \epsilon\left\langle\left(-\Delta_{\Gamma}\right)^{1 / 4} \delta_{t}^{c},\left(-\Delta_{\Gamma}\right)^{1 / 4} \delta^{c}\right\rangle+2\left\langle\left(-\Delta_{\Gamma}\right)^{1 / 4} u^{c},\left(-\Delta_{\Gamma}\right)^{1 / 4} \delta_{t}^{c}\right\rangle \\
& \left.+2 \epsilon\left\langle\left(-\Delta_{\Gamma}\right)^{1 / 4} u^{c},\left(-\Delta_{\Gamma}\right)^{1 / 4} \delta^{c}\right\rangle\right\}+\epsilon\left\|\left(-\Delta_{\Gamma}\right)^{1 / 4} \delta^{c}\right\|_{L^{2}(\Gamma)}^{2} \\
& +(\nu-\epsilon)\left\|\left(-\Delta_{\Gamma}\right)^{1 / 4} \delta_{t}^{c}\right\|_{L^{2}(\Gamma)}^{2}=-\left\langle\left(-\Delta_{\Gamma}\right)^{1 / 4} u^{c},\left(-\Delta_{\Gamma}\right)^{1 / 4} \delta^{c}\right\rangle \\
& -(\nu-\epsilon)\left\langle\left(-\Delta_{\Gamma}\right)^{1 / 4} u^{c},\left(-\Delta_{\Gamma}\right)^{1 / 4} \delta_{t}^{c}\right\rangle .
\end{aligned}
$$

We now introduce the functional

$$
\begin{aligned}
& \Lambda_{2}:=(1+\epsilon \nu)\left\|\left(-\Delta_{\Gamma}\right)^{1 / 4} \delta^{c}\right\|_{L^{2}(\Gamma)}^{2}+\left\|\left(-\Delta_{\Gamma}\right)^{1 / 4} \delta_{t}^{c}\right\|_{L^{2}(\Gamma)}^{2}+\left\|\left(-\Delta_{\Gamma}\right)^{1 / 4} u^{c}\right\|_{L^{2}(\Gamma)}^{2} \\
& +2 \epsilon\left\langle\left(-\Delta_{\Gamma}\right)^{1 / 4} \delta_{t}^{c},\left(-\Delta_{\Gamma}\right)^{1 / 4} \delta^{c}\right\rangle+2\left\langle\left(-\Delta_{\Gamma}\right)^{1 / 4} u^{c},\left(-\Delta_{\Gamma}\right)^{1 / 4} \delta_{t}^{c}\right\rangle \\
& +2 \epsilon\left\langle\left(-\Delta_{\Gamma}\right)^{1 / 4} u^{c},\left(-\Delta_{\Gamma}\right)^{1 / 4} \delta^{c}\right\rangle .
\end{aligned}
$$


By means of the trace theorem (see [22, Theorem 9.4, Chapter 1]), we have

$$
\left\|\left(-\Delta_{\Gamma}\right)^{1 / 4} u^{c}\right\|_{L^{2}(\Gamma)} \leq\left\|u^{c}\right\|_{H^{1 / 2}(\Gamma)} \leq c\left\|u^{c}\right\|_{1} \leq c k
$$

and therefore it is easy to prove that, for $\epsilon \in(0,1 / 4]$, the functional $\Lambda_{2}$ satisfies the inequality

$$
\begin{aligned}
& \frac{1}{4}\left(\left\|\left(-\Delta_{\Gamma}\right)^{1 / 4} \delta^{c}\right\|_{L^{2}(\Gamma)}^{2}+\left\|\left(-\Delta_{\Gamma}\right)^{1 / 4} \delta_{t}^{c}\right\|_{L^{2}(\Gamma)}^{2}\right)-c k^{2} \leq \Lambda_{2} \\
& \leq(2+\nu)\left(\left\|\left(-\Delta_{\Gamma}\right)^{1 / 4} \delta^{c}\right\|_{L^{2}(\Gamma)}^{2}+\left\|\left(-\Delta_{\Gamma}\right)^{1 / 4} \delta_{t}^{c}\right\|_{L^{2}(\Gamma)}^{2}\right)+c k^{2} .
\end{aligned}
$$

From identity (5.38) we obtain

$$
\begin{aligned}
& \frac{d \Lambda_{2}}{d t}+2 \epsilon\left\|\left(-\Delta_{\Gamma}\right)^{1 / 4} \delta^{c}\right\|_{L^{2}(\Gamma)}^{2}+2(\nu-\epsilon)\left\|\left(-\Delta_{\Gamma}\right)^{1 / 4} \delta_{t}^{c}\right\|_{L^{2}(\Gamma)}^{2} \\
& \leq\left(\left\|\left(-\Delta_{\Gamma}\right)^{1 / 4} \delta^{c}\right\|_{L^{2}(\Gamma)}+\nu\left\|\left(-\Delta_{\Gamma}\right)^{1 / 4} \delta_{t}^{c}\right\|_{L^{2}(\Gamma)}\right)\left\|\left(-\Delta_{\Gamma}\right)^{1 / 4} u^{c}\right\|_{L^{2}(\Gamma)} \\
& \leq \epsilon\left(\left\|\left(-\Delta_{\Gamma}\right)^{1 / 4} \delta^{c}\right\|_{L^{2}(\Gamma)}^{2}+\left\|\left(-\Delta_{\Gamma}\right)^{1 / 4} \delta_{t}^{c}\right\|_{L^{2}(\Gamma)}^{2}\right)+\frac{c k^{2}}{\epsilon}
\end{aligned}
$$

Therefore, by taking $0<\epsilon \leq \epsilon_{0}(\nu):=\min \{1 / 4, \nu / 2\}$, from (5.41) we get

$$
\frac{d \Lambda_{2}}{d t}+\epsilon\left(\left\|\left(-\Delta_{\Gamma}\right)^{1 / 4} \delta^{c}\right\|_{L^{2}(\Gamma)}^{2}+\left\|\left(-\Delta_{\Gamma}\right)^{1 / 4} \delta_{t}^{c}\right\|_{L^{2}(\Gamma)}^{2}\right) \leq \frac{c k^{2}}{\epsilon},
$$

and, by using (5.40), we are led to the following differential inequality

$$
\frac{d \Lambda_{2}}{d t}+\epsilon_{0}^{\prime}(\nu) \Lambda_{2} \leq b_{8}
$$

where $\epsilon_{0}^{\prime}(\nu)=\epsilon_{0}(\nu) /(2+\nu)$ and $b_{8}=c \epsilon_{0}(\nu) k^{2} /(2+\nu)+c k^{2} / \epsilon_{0}(\nu)$. The standard Gronwall lemma and (5.40) finally give (notice that $\Lambda_{2}(0)=0$ )

$$
\left\|\delta^{c}(t)\right\|_{H^{1 / 2}(\Gamma)}+\left\|\delta_{t}^{c}(t)\right\|_{H^{1 / 2}(\Gamma)} \leq b_{9}
$$

We now observe that, by virtue of an $H^{2}$-elliptic regularity estimate (obtained by combination of Theorem 2.5.1 and 2.5.3 in [22]), we can write

$$
\begin{aligned}
& \left\|u^{c}\right\|_{2} \leq c\left(\left\|\Delta u^{c}\right\|+\left\|u^{c}\right\|_{1}+\left\|\partial_{\mathbf{n}} u^{c}\right\|_{H^{1 / 2}(\Gamma)}\right) \\
& \leq c\left(\left\|\Delta u^{c}\right\|+\left\|u^{c}\right\|_{1}+\left\|\delta_{t}^{c}\right\|_{H^{1 / 2}(\Gamma)}\right) \leq b_{10} .
\end{aligned}
$$

The thesis finally follows from (5.44), (5.37) and (5.43).

Proof of Theorem 4. Let us take $\mathcal{B}_{1}(\omega, \nu)=\left\{w \in \mathcal{H}_{1}:\|w\|_{\mathcal{H}_{1}} \leq b_{\omega, \nu}\right\}$, with $b_{\omega, \nu}$ as in Lemma 8. The thesis is then an immediate consequence of Lemma 7 and of Lemma 8 (the constant $c_{\omega, \nu}$ in (5.1) being the same as in (5.8)). 
Remark 4. We have not written explicitly the dependence of the radius $b_{\omega, \nu}$ of $\mathcal{B}_{1}$ on $\omega$ and $\nu$, since its expression would turn out rather cumbersome. Nevertheless, by using the (continuous) $\omega, \nu$-dependence of the constants $b_{i}$ in the proof of Lemma 8, and also by means of Lemma 3, one would see that, for $\omega_{0}$ and $\nu_{0}>0$ fixed arbitrary, we have $b_{\omega, \nu} \rightarrow+\infty$ as $(\omega, \nu) \rightarrow\left(\omega_{0}, 0\right)$, or as $(\omega, \nu) \rightarrow\left(0, \nu_{0}\right)$, or as $|\omega|+|\nu| \rightarrow+\infty$. This implies the existence of a couple $\left(\omega_{*}, \nu_{*}\right)$ where the radius of the regular attracting set $\mathcal{B}_{1}(\omega, \nu)$ attains its minimum.

\section{$6 \quad$ Exponential attractors}

We conclude the paper with the section containing a result on the existence of an exponential attractor, namely, of a compact set $\mathcal{M}=\mathcal{M}_{\omega, \nu} \subset \mathcal{H}$, positively invariant for $S(t)$, of finite fractal dimension, and satisfying the following exponential attraction property:

There exists an increasing function $J:[0,+\infty) \rightarrow[0,+\infty)$ and $\kappa>0$ such that, for every $R>0$ and for every set $\mathcal{B} \subset \mathcal{H}$ with $\sup _{w_{0} \in \mathcal{B}}\left\|w_{0}\right\|_{0} \leq R$ there holds

$$
\operatorname{dist}_{\mathcal{H}}(S(t) \mathcal{B}, \mathcal{M}) \leq J(R) e^{-\kappa t}
$$

for all $t>0$.

Theorem 5. Let (1.2)-(1.5) hold. Then the semigroup $S(t)$ on $\mathcal{H}$ associated with (1.1) possesses an exponential attractor $\mathcal{M}=\mathcal{M}_{\omega, \nu}$.

For the proof of Theorem 5 we make use of the abstract result on the existence of an exponential attractor due to Efendiev, Miranville, Zelik [10]. In order to follow this approach it is firstly useful to construct a bounded absorbing set in $\mathcal{H}_{1}$. This is done in the following lemma.

Lemma 9. Let (1.2)-(1.5) hold. Then, there exist constants $K_{1}, K_{2}>0$, with $K_{1}=K_{1}\left(\left\|w_{0}\right\|_{\mathcal{H}_{1}},\left\|w_{0}\right\|_{\mathcal{H}}\right)$ and $K_{2}=K_{2}\left(\left\|w_{0}\right\|_{\mathcal{H}}\right)$ depending increasingly and continuously on $\left\|w_{0}\right\|_{\mathcal{H}_{1}}$ and $\left\|w_{0}\right\|_{\mathcal{H}}$, respectively (and also on $\omega, \nu$ ), and there exists $\widehat{\epsilon}>0$ (depending on $\omega, \nu$ in the form $\widehat{\epsilon} \sim J(\omega, \nu)$ ) such that

$$
\|w(t)\|_{\mathcal{H}_{1}} \leq K_{1} e^{-\widehat{\epsilon} t}+K_{2},
$$

for all $t \geq 0$.

Corollary 4. The ball in $\mathcal{H}_{1}$ given by $\mathcal{B}_{0}^{(1)}:=\left\{w \in \mathcal{H}_{1}:\|w\|_{\mathcal{H}_{1}} \leq 2 K_{2}\left(R_{0}\right)\right\}$ is a bounded absorbing set in $\mathcal{H}_{1}$ for the semigroup $S(t): \mathcal{H}_{1} \rightarrow \mathcal{H}_{1}$.

Remark 5. It can be proved that, under assumptions (1.2)-(1.4), $S(t)$ is also a strongly continuous semigroup on the phase space $\mathcal{H}_{1}$. 
Proof of Lemma 9. We can argue exactly as in the proof of Lemma 8 applied to system (1.1) written in the form (5.3) (with $w$ in place of $w^{c}, \theta=l$ and an initial condition $\left.w(0)=w_{0} \in \mathcal{H}_{1}\right)$.

We again get the differential inequalities (5.34) and (5.42) for the functionals $\Lambda_{1}$ and $\Lambda_{2}$, respectively. The only difference is that now we have $\Lambda_{1}(0) \leq C_{\omega, \nu}\left(\left\|w_{0}\right\|_{\mathcal{H}_{1}},\left\|w_{0}\right\|_{\mathcal{H}}\right)$ and $\Lambda_{2}(0) \leq C_{\omega, \nu}\left(\left\|w_{0}\right\|_{\mathcal{H}_{1}},\left\|w_{0}\right\|_{\mathcal{H}}\right)$

By using the Gronwall lemmas and the elliptic regularity estimate in $H^{2}$, as in the proof of Lemma 8, we deduce (6.2).

In the previous section we proved the existence of a bounded subset $\mathcal{B}_{1}$ of $\mathcal{H}_{1}$ which attracts the bounded subsets of $\mathcal{H}$ exponentially fast (Theorem 4). Due to the existence of a bounded absorbing set in $\mathcal{H}_{1}$, it is easy to see that, up to possibly enlarging $\mathcal{B}_{1}$, there is a time $t_{1} \geq 0$ such that $S(t) \mathcal{B}_{1} \subset \mathcal{B}_{1}$, for all $t \geq t_{1}$.

We now appeal to the following abstract result [10] (see also [27]).

Lemma 10. Assume that there exists a time $t^{*} \geq t_{1}$ such that

(i) the map $(t, z) \mapsto S(t) z:\left[t^{*}, 2 t^{*}\right] \times \mathcal{B}_{1} \rightarrow \mathcal{B}_{1}$ is Lipschitz continuous when $\mathcal{B}_{1}$ is endowed with the topology inherited from $\mathcal{H}$;

(ii) the map $S\left(t^{*}\right): \mathcal{B}_{1} \rightarrow \mathcal{B}_{1}$ admits a decomposition of the form

$$
S\left(t^{*}\right)=S_{d}+S_{c}, \quad S_{d}: \mathcal{B}_{1} \rightarrow \mathcal{H}, \quad S_{c}: \mathcal{B}_{1} \rightarrow \mathcal{H}_{1}
$$

where $S_{d}$ and $S_{c}$ satisfy the conditions

$$
\begin{gathered}
\left\|S_{d}\left(w_{2}\right)-S_{d}\left(w_{1}\right)\right\|_{\mathcal{H}} \leq \gamma_{*}\left\|w_{2}-w_{1}\right\|_{\mathcal{H}}, \\
\left\|S_{c}\left(w_{2}\right)-S_{c}\left(w_{1}\right)\right\|_{\mathcal{H}_{1}} \leq \Gamma_{*}\left\|w_{2}-w_{1}\right\|_{\mathcal{H}}, \\
\text { for every } w_{1}, w_{2} \in \mathcal{B}_{1} \text { and for some } \gamma_{*} \in(0,1 / 2) \text { and } \Gamma_{*} \geq 0 .
\end{gathered}
$$

Then there exists a compact set $\mathcal{M} \subset \mathcal{B}_{1}$, positively invariant for $S(t)$ and of finite fractal dimension in $\mathcal{H}$, such that

$$
\operatorname{dist}_{\mathcal{H}}\left(S(t) \mathcal{B}_{1}, \mathcal{M}\right) \leq M e^{-\lambda_{0} t}
$$

for some $\lambda_{0}>0$ and $M \geq 0$, for all $t>0$.

In the following lemmas we check the assumptions of Lemma 10.

Lemma 11. Assumption (i) of Lemma 10 holds true. 
Proof. We first observe that we have

$$
\left\|w_{t}(t)\right\|_{\mathcal{H}} \leq c_{\omega, \nu}
$$

for every $w_{0} \in \mathcal{B}_{1}$ and every $t \geq 0$. Indeed, by differentiating the first equation of system (1.1), we can argue as in the first part of the proof of Lemma 8 thus yielding a control of the form (5.37) (with $u$ instead of $u^{c}$ ). Furthermore, by noting that we obviously have $\left\|\delta_{t t}\right\|_{L^{2}(\Gamma)} \leq \nu\left\|\delta_{t}\right\|_{L^{2}(\Gamma)}+\|\delta\|_{L^{2}(\Gamma)}+c_{t}\left\|u_{t}\right\|_{1} \leq$ $c_{\omega, \nu}$, we get (6.4). Now, let $w_{1}, w_{2} \in \mathcal{B}_{1}$ and $t_{1}, t_{2} \in\left[t^{*}, 2 t^{*}\right]$. We write

$$
\left\|S\left(t_{2}\right) w_{2}-S\left(t_{1}\right) w_{1}\right\|_{\mathcal{H}} \leq\left\|S\left(t_{2}\right) w_{2}-S\left(t_{2}\right) w_{1}\right\|_{\mathcal{H}}+\left\|S\left(t_{2}\right) w_{1}-S\left(t_{1}\right) w_{1}\right\|_{\mathcal{H}} .
$$

Then, by using the continuous dependence estimate (2.3) and (6.4), we conclude the proof.

Lemma 12. Assumption (ii) of Lemma 10 holds true.

Proof. We take $w_{01}, w_{02} \in \mathcal{B}_{1}$ and consider the corresponding trajectories, namely $w^{1}=\left(u^{1}, u_{t}^{1}, \delta^{1}, \delta_{t}^{1}\right)$ and $w^{2}=\left(u^{2}, u_{t}^{2}, \delta^{2}, \delta_{t}^{2}\right)$.

Setting $\bar{w}:=w^{2}-w^{1}$ and $\bar{w}_{0}:=w_{02}-w_{01}$, we decompose $\bar{w}$ as $\bar{w}=$ $\bar{w}^{d}+\bar{w}^{c}=:\left(\bar{u}^{d}, \bar{u}_{t}^{d}, \bar{\delta}^{d}, \bar{\delta}_{t}^{d}\right)+\left(\bar{u}^{c}, \bar{u}_{t}^{c}, \bar{\delta}^{c}, \bar{\delta}_{t}^{c}\right)$, where le components of $\bar{w}^{d}$ and $\bar{w}^{c}$ solve, respectively

$$
\left\{\begin{array}{l}
\bar{u}_{t t}^{d}+\omega \bar{u}_{t}^{d}-\Delta \bar{u}^{d}+\bar{u}^{d}=0 \\
\bar{\delta}_{t t}^{d}+\nu \bar{\delta}_{t}^{d}+\bar{\delta}^{d}=-\bar{u}_{t}^{d} \\
\bar{\delta}_{t}^{d}=\partial_{\mathbf{n}} \bar{u}^{d} \\
\bar{w}^{d}(0)=\bar{w}_{0}
\end{array}\right.
$$

and

$$
\left\{\begin{array}{l}
\bar{u}_{t t}^{c}+\omega \bar{u}_{t}^{c}-\Delta \bar{u}^{c}+\bar{u}^{c}=f\left(u^{1}\right)-f\left(u^{2}\right) \\
\bar{\delta}_{t t}^{c}+\nu \bar{\delta}_{t}^{c}+\bar{\delta}^{c}=-\bar{u}_{t}^{c} \\
\bar{\delta}_{t}^{c}=\partial_{\mathbf{n}} \bar{u}^{c} \\
\bar{w}^{c}(0)=0
\end{array}\right.
$$

System (6.5) is linear and it is not difficult to show, by means of standard arguments (see the proof of Lemma 7), that the corresponding solution fulfills

$$
\left\|\bar{w}^{d}(t)\right\|_{\mathcal{H}} \leq c\left\|\bar{w}_{0}\right\|_{\mathcal{H}} e^{-c J(\omega, \nu) t}
$$

for all $t \geq 0$, and for some $c$ independent of $\omega, \nu$. Hence, if we fix $t^{*} \geq$ $(c J)^{-1} \log (4 c)$, we fulfill the first part of assumption (ii) of Lemma 10. 
Furthermore, by multiplying $(6.6)_{1}$ in $L^{2}(\Omega)$ by $-\Delta \bar{u}_{t}^{c}$ we obtain

$$
\begin{aligned}
& \frac{1}{2} \frac{d}{d t}\left\{\left\|\Delta \bar{u}^{c}\right\|^{2}+\left\|\nabla \bar{u}^{c}\right\|^{2}+\left\|\nabla \bar{u}_{t}^{c}\right\|^{2}+\left\|\bar{u}^{c}\right\|_{L^{2}(\Gamma)}^{2}+\left\|\bar{u}_{t}^{c}\right\|_{L^{2}(\Gamma)}^{2}\right. \\
& \left.+2\left\langle\nu \bar{\delta}_{t}^{c}+\bar{\delta}^{c}, \bar{u}_{t}^{c}\right\rangle\right\}+\omega\left\|\nabla \bar{u}_{t}^{c}\right\|^{2}+(\omega+\nu)\left\|\bar{u}_{t}^{c}\right\|_{L^{2}(\Gamma)}^{2} \\
& =-\left\langle\left(\nu^{2}+\omega \nu-1\right) \bar{\delta}_{t}^{c}+(\omega+\nu) \bar{\delta}^{c}, \bar{u}_{t}^{c}\right\rangle-\left\langle\nu \bar{\delta}_{t}^{c}+\bar{\delta}^{c}, \bar{u}^{c}\right\rangle \\
& +\left(\left(f^{\prime}\left(u^{1}\right)-f^{\prime}\left(u^{2}\right)\right) \nabla u^{1}, \nabla \bar{u}_{t}^{c}\right)-\left(f^{\prime}\left(u^{2}\right) \nabla \bar{u}, \nabla \bar{u}_{t}^{c}\right) \\
& +\left\langle f\left(u^{1}\right)-f\left(u^{2}\right), \nu \bar{\delta}_{t}^{c}+\bar{\delta}^{c}+\bar{u}_{t}^{c}\right\rangle .
\end{aligned}
$$

Now, due to the continuous dependence estimate (2.3) and to (6.7), we have $\left\|\bar{\delta}^{c}\right\|_{L^{2}(\Gamma)} \leq\|\bar{\delta}\|_{L^{2}(\Gamma)}+\left\|\bar{\delta}^{d}\right\|_{L^{2}(\Gamma)} \leq c_{\omega, \nu}\left(t^{*}\right)\left\|\bar{w}_{0}\right\|_{\mathcal{H}}$, for all $t \in\left[0, t^{*}\right]$, and a similar estimate holds for $\left\|\bar{\delta}_{t}^{c}\right\|_{L^{2}(\Gamma)},\left\|\bar{u}^{c}\right\|_{1}$ and $\left\|\bar{u}_{t}^{c}\right\|$.

Therefore, for all $t \in\left[0, t^{*}\right]$ we have

$$
\begin{aligned}
& \left|\left\langle\left(\nu^{2}+\omega \nu-1\right) \bar{\delta}_{t}^{c}+(\omega+\nu) \bar{\delta}^{c}, \bar{u}_{t}^{c}\right\rangle\right| \leq c_{\omega, \nu}\left(t^{*}\right)\left\|\bar{w}_{0}\right\|_{\mathcal{H}}\left\|\bar{u}_{t}^{c}\right\|_{1} \\
& \leq \frac{\omega}{8}\left\|\nabla \bar{u}_{t}^{c}\right\|^{2}+c_{\omega, \nu}\left(t^{*}\right)\left\|\bar{w}_{0}\right\|_{\mathcal{H}}^{2}, \\
& \left|\left\langle\nu \bar{\delta}_{t}^{c}+\bar{\delta}^{c}, \bar{u}^{c}\right\rangle\right| \leq c_{\omega, \nu}\left(t^{*}\right)\left\|\bar{w}_{0}\right\|_{\mathcal{H}}^{2} .
\end{aligned}
$$

Furthermore, by (1.7) and Lemma 9 , for all $t \in\left[0, t^{*}\right]$ we have

$$
\begin{aligned}
& \left|\left(\left(f^{\prime}\left(u^{1}\right)-f^{\prime}\left(u^{2}\right)\right) \nabla u^{1}, \nabla \bar{u}_{t}^{c}\right)\right| \leq c\left(1+\left\|u^{1}\right\|_{1}+\left\|u^{2}\right\|_{1}\right)\|\bar{u}\|_{1}\left\|u^{1}\right\|_{2}\left\|\nabla \bar{u}_{t}^{c}\right\| \\
& \leq c_{\omega, \nu}\left(t^{*}\right)\left\|\bar{w}_{0}\right\|\left\|_{\mathcal{H}}\right\| \nabla \bar{u}_{t}^{c}\left\|\leq \frac{\omega}{8}\right\| \nabla \bar{u}_{t}^{c}\left\|^{2}+c_{\omega, \nu}\left(t^{*}\right)\right\| \bar{w}_{0} \|_{\mathcal{H}}^{2}, \\
& \left|\left(f^{\prime}\left(u^{2}\right) \nabla \bar{u}, \nabla \bar{u}_{t}^{c}\right)\right| \leq c\left(1+\left\|u^{2}\right\|_{2}^{2}\right)\|\nabla \bar{u}\|\left\|\nabla \bar{u}_{t}^{c}\right\| \leq c_{\omega, \nu}\left(t^{*}\right)\left\|\bar{w}_{0}\right\|_{\mathcal{H}}\left\|\nabla \bar{u}_{t}^{c}\right\| \\
& \leq \frac{\omega}{8}\left\|\nabla \bar{u}_{t}^{c}\right\|^{2}+c_{\omega, \nu}\left(t^{*}\right)\left\|\bar{w}_{0}\right\|_{\mathcal{H}}^{2}, \\
& \left|\left\langle f\left(u^{1}\right)-f\left(u^{2}\right), \nu \bar{\delta}_{t}^{c}+\bar{\delta}^{c}+\bar{u}_{t}^{c}\right\rangle\right| \\
& \leq c_{\omega, \nu}\left(t^{*}\right)\left(1+\left\|u^{1}\right\|_{C^{0}(\Gamma)}^{2}+\left\|u^{2}\right\|_{C^{0}(\Gamma)}^{2}\right)\|\bar{u}\|_{L^{2}(\Gamma)}\left(\left\|\bar{w}_{0}\right\|_{\mathcal{H}}+\left\|\bar{u}_{t}^{c}\right\|_{1}\right) \\
& \leq \frac{\omega}{8}\left\|\nabla \bar{u}_{t}^{c}\right\|^{2}+c_{\omega, \nu}\left(t^{*}\right)\left\|\bar{w}_{0}\right\|_{\mathcal{H}}^{2},
\end{aligned}
$$

where in (6.12) and (6.13) we have also used the continuous embedding $H^{2}(\Omega) \hookrightarrow C^{0}(\bar{\Omega})$. Plugging (6.9)-(6.13) into (6.8), we obtain

$$
\begin{aligned}
& \frac{d}{d t}\left\{\left\|\Delta \bar{u}^{c}\right\|^{2}+\left\|\nabla \bar{u}^{c}\right\|^{2}+\left\|\nabla \bar{u}_{t}^{c}\right\|^{2}+\left\|\bar{u}^{c}\right\|_{L^{2}(\Gamma)}^{2}+\left\|\bar{u}_{t}^{c}\right\|_{L^{2}(\Gamma)}^{2}\right. \\
& \left.+2\left\langle\nu \bar{\delta}_{t}^{c}+\bar{\delta}^{c}, \bar{u}_{t}^{c}\right\rangle\right\} \leq c_{\omega, \nu}\left(t^{*}\right)\left\|\bar{w}_{0}\right\|_{\mathcal{H}}^{2} .
\end{aligned}
$$

By integrating (6.14) between 0 and $t^{*}$ we are led to the following estimate

$$
\left\|\Delta \bar{u}^{c}\left(t^{*}\right)\right\|+\left\|\nabla \bar{u}_{t}^{c}\left(t^{*}\right)\right\| \leq c_{\omega, \nu}\left(t^{*}\right)\left\|\bar{w}_{0}\right\|_{\mathcal{H}} .
$$


Let us now consider the second equation in (6.6). By multiplying it in $L^{2}(\Gamma)$ by $\left(-\Delta_{\Gamma}\right)^{1 / 2} \bar{\delta}_{t}^{c}$ we get

$$
\begin{aligned}
& \frac{1}{2} \frac{d}{d t}\left\{\left\|\left(-\Delta_{\Gamma}\right)^{1 / 4} \bar{\delta}^{c}\right\|_{L^{2}(\Gamma)}^{2}+\left\|\left(-\Delta_{\Gamma}\right)^{1 / 4} \bar{\delta}_{t}^{c}\right\|_{L^{2}(\Gamma)}^{2}+\left\|\left(-\Delta_{\Gamma}\right)^{1 / 4} \bar{u}^{c}\right\|_{L^{2}(\Gamma)}^{2}\right. \\
& \left.+2\left\langle\left(-\Delta_{\Gamma}\right)^{1 / 4} \bar{u}^{c},\left(-\Delta_{\Gamma}\right)^{1 / 4} \bar{\delta}_{t}^{c}\right\rangle\right\}+\nu\left\|\left(-\Delta_{\Gamma}\right)^{1 / 4} \bar{\delta}_{t}^{c}\right\|_{L^{2}(\Gamma)}^{2} \\
& =-\nu\left\langle\left(-\Delta_{\Gamma}\right)^{1 / 4} \bar{u}^{c},\left(-\Delta_{\Gamma}\right)^{1 / 4} \bar{\delta}_{t}^{c}\right\rangle-\left\langle\left(-\Delta_{\Gamma}\right)^{1 / 4} \bar{u}^{c},\left(-\Delta_{\Gamma}\right)^{1 / 4} \bar{\delta}^{c}\right\rangle \\
& \leq c_{\nu}\left(\left\|\left(-\Delta_{\Gamma}\right)^{1 / 4} \bar{\delta}_{t}^{c}\right\|_{L^{2}(\Gamma)}+\left\|\left(-\Delta_{\Gamma}\right)^{1 / 4} \bar{\delta}^{c}\right\|_{L^{2}(\Gamma)}\right)\left\|\left(-\Delta_{\Gamma}\right)^{1 / 4} \bar{u}^{c}\right\|_{L^{2}(\Gamma)} \\
& \leq c_{\omega, \nu}\left(t^{*}\right)\left(\left\|\left(-\Delta_{\Gamma}\right)^{1 / 4} \bar{\delta}_{t}^{c}\right\|_{L^{2}(\Gamma)}+\left\|\left(-\Delta_{\Gamma}\right)^{1 / 4} \bar{\delta}^{c}\right\|_{L^{2}(\Gamma)}\right)\left\|\bar{w}_{0}\right\|_{\mathcal{H}}
\end{aligned}
$$

where, in the last inequality, we have made use of the estimate

$$
\left\|\left(-\Delta_{\Gamma}\right)^{1 / 4} \bar{u}^{c}\right\|_{L^{2}(\Gamma)} \leq\left\|\bar{u}^{c}\right\|_{H^{1 / 2}(\Gamma)} \leq\left\|\bar{u}^{c}\right\|_{1} \leq c_{\omega, \nu}\left(t^{*}\right)\left\|\bar{w}_{0}\right\|_{\mathcal{H}}
$$

for every $t \in\left[0, t^{*}\right]$. From (6.16), by means of the Young inequality and of the standard Gronwall lemma, it is not difficult to deduce the control

$$
\left\|\bar{\delta}^{c}\left(t^{*}\right)\right\|_{H^{1 / 2}(\Gamma)}+\left\|\bar{\delta}_{t}^{c}\left(t^{*}\right)\right\|_{H^{1 / 2}(\Gamma)} \leq c_{\omega, \nu}\left(t^{*}\right)\left\|\bar{w}_{0}\right\|_{\mathcal{H}} .
$$

Now, from $(6.15),(6.17)$, and the $H^{2}$-elliptic regularity estimate (see (5.44)) we immediately get

$$
\left\|\bar{w}^{c}\left(t^{*}\right)\right\|_{\mathcal{H}_{1}} \leq c_{\omega, \nu}\left\|\bar{w}_{0}\right\|_{\mathcal{H}}
$$

The proof is then completed.

Proof of Theorem 5. By combining Lemma 10, Lemma 11 and Lemma 12, we deduce the existence of a compact invariant subset $\mathcal{M}_{\omega, \nu} \subset \mathcal{B}_{1} \subset \mathcal{H}_{1}$ such that (6.3) holds (with $M$ and $\lambda_{0}$ depending on $\omega, \nu$ ).

In order to show that $\mathcal{M}_{\omega, \nu}$ is an exponential attractor it remains to prove that the basin of attraction of $\mathcal{M}_{\omega, \nu}$ is the whole phase space $\mathcal{H}$. This can be easily accomplished by means of $(5.1),(6.3),(2.3)$ and by appealing to the transitivity property of the exponential attraction (see [11, Theorem $5.1])$.

Acknowledgements. I would like to express my thanks to Professor Maurizio Grasselli for having submitted this problem to my attention, for many remarks and very fruitful discussions.

\section{References}

[1] J. M. Ball, Global attractors for damped semilinear wave equations, Discrete Contin. Dyn. Syst., 10 (2004), 31-52. 
[2] J. M. Ball, Strongly continuous semigroups, weak solutions, and the variation of constants formula, Proc. Amer. Math. Soc., 64 (1977), 370-373.

[3] J. T. Beale and S. I. Rosencrans, Acoustic boundary conditions, Bull. Amer. Math. Soc., 80 (1974), 1276-1278.

[4] J. T. Beale, Spectral properties of an acoustic boundary condition, Indiana Univ. Math. J., 25 (1976), 895-917.

[5] J. T. Beale, Acoustic scattering from locally reacting surfaces, Indiana Univ. Math. J., 26 (1977), 199-222.

[6] V. Belleri and V. Pata, Attractors for semilinear strongly damped wave equation on $\mathbb{R}^{n}$, Discrete Contin. Dyn. Syst., 7 (2001), 719-735.

[7] V. Casarino, K. J. Engel, R. Nagel and G. Nickel, A semigroup approach to boundary feedback systems, Integral Equations Operator Theory, 47 (2003), 289-306.

[8] V. Casarino, K. J. Engel, G. Nickel and S. Piazzera, Decoupling techniques for wave equations with dynamic boundary conditions, Discrete Contin. Dyn. Syst. Ser. B, 12 (2005), 761-772.

[9] M. Conti and V. Pata, Weakly dissipative semilinear equations of viscoelasticity, Commun. Pure Appl. Anal., 4 (2005), 705-720.

[10] M. Efendiev, A. Miranville and S. Zelik, Exponential attractors for a nonlinear reaction-diffusion system in $\mathbb{R}^{3}$, C. R. Acad. Sci. Paris Sr. I Math., 330 (2000), 713-718.

[11] P. Fabrie, C. Galusinski, A. Miranville and S. Zelik, Uniform exponential attractors for a singularly perturbed damped wave equation, Discrete Contin. Dyn. Syst., 10 (2004), 211-238.

[12] S. Frigeri, Asymptotic behavior of a hyperbolic system arising in ferroelectricity, Commun. Pure Appl. Anal., 7 (2008), 1393-1414.

[13] S. Frigeri, Long time behavior of some semilinear hyperbolic systems, Ph. D. Thesis, in preparation.

[14] C. L. Frota and J. A. Goldstein, Some nonlinear wave equations with acoustic boundary conditions, J. Differential Equations, 164 (2000), 92-109. 
[15] C. G. Gal, G. R. Goldstein and J. A. Goldstein, Oscillatory boundary conditions for acoustic wave equations, J. Evol. Equ., 3 (2003), 623-636.

[16] S. Gatti, M. Grasselli, A. Miranville and V. Pata, On the hyperbolic relaxation of the one-dimensional Cahn-Hilliard equation, J. Math. Anal. Appl., 312 (2006), 230-247.

[17] J. M. Ghidaglia, A note on the strong convergence towards attractors for damped forced KdV equations, J. Differential Equations, 110 (1994), 356-359.

[18] O. Goubet, Regularity of the attractor for the weakly damped nonlinear Schrödinger equation, Appl. Anal., 60 (1996), 99-119.

[19] O. Goubet and I. Moise, Attractor for dissipative Zakharov system, Nonlinear Anal., 31 (1998), 823-847.

[20] M. Grasselli, A. Miranville, V. Pata and S. Zelik, Well-posedness and long time behavior of a parabolic-hyperbolic phase-field system with singular potentials, Math. Nachr., 280 (2007), 1475-1509.

[21] M. Grasselli and V. Pata, Asymptotic behavior of a parabolic-hyperbolic system, Commun. Pure Appl. Anal., 3 (2004), 849-881.

[22] J.-L. Lions and E. Magenes, Non-Homogeneous Boundary Value Problems and Applications, Springer-Verlag, New-York, 1972.

[23] I. Moise and R. Rosa, On the regularity of the global attractor of a weakly damped forced Korteweg-de Vries equation, Adv. Differential Equation, 2 (1997), 257-296.

[24] I. Moise, R. Rosa and X. Wang, Attractors for non-compact semigroups via energy equation, Nonlinearity, 11 (1998), 1369-1393.

[25] P. M. Morse and K. U. Ingard, Theoretical acoustics, McGraw-Hill, New York, 1968.

[26] D. Mugnolo, Abstract wave equations with acoustic boundary conditions, Math. Nachr., 279 (2006), 299-318.

[27] V. Pata and M. Squassina, On the strongly damped wave equation, Comm. Math. Phys., 253 (2005), 511-533.

[28] V. Pata and S. Zelik, A remark on the damped wave equation, Commun. Pure Appl. Anal., 5 (2006), 609-614. 
[29] R. Rosa, The global attractor for the 2d Navier-Stokes flow on some unbounded domains, Nonlinear Anal., 32 (1998), 71-85.

[30] X. Wang, An energy equation for the weakly damped driven nonlinear Schrödinger equations and its applications, Phys. D, 88 (1995), 167175.

[31] P. J. Yeoul and K. J. Ae, Some nonlinear wave equations with nonlinear memory source term and acoustic boundary conditions, Numer. Funct. Anal. Optim., 27 (2006), 889-903.

[32] S. Zelik, Asymptotic regularity of solutions of singularly perturbed damped wave equations with supercritical nonlinearities, Discrete Contin. Dyn. Syst., 11 (2004), 351-392.

[33] S. Zheng, Nonlinear Evolution Equations, Pitman Monographs and Surveys in Pure and Applied Mathematics, 133, CHAPMAN and HALL/CRC, Boca Raton, Florida, 2004. 